Portland State University

PDXScholar

Dissertations and Theses

Dissertations and Theses

Summer 10-4-2013

\title{
Optics and Spectroscopy in Massive Electrodynamic Theory
}

Adam Caccavano

Portland State University

Follow this and additional works at: https://pdxscholar.library.pdx.edu/open_access_etds

Part of the Electromagnetics and Photonics Commons, and the Optics Commons Let us know how access to this document benefits you.

Recommended Citation

Caccavano, Adam, "Optics and Spectroscopy in Massive Electrodynamic Theory" (2013). Dissertations and Theses. Paper 1485.

https://doi.org/10.15760/etd.1484

This Thesis is brought to you for free and open access. It has been accepted for inclusion in Dissertations and Theses by an authorized administrator of PDXScholar. Please contact us if we can make this document more accessible: pdxscholar@pdx.edu. 
Optics and Spectroscopy in Massive Electrodynamic Theory

by

Adam Caccavano

A thesis submitted in partial fulfillment of the requirements for the degree of

\author{
Master of Science \\ in \\ Physics
}

Thesis Committee:

Pui-Tak Leung

Erik Sanchez

Jack Straton

Portland State University

2013 


\section{(C) 2013 Adam Caccavano}




\section{ABSTRACT}

The kinematics and dynamics for plane wave optics are derived for a massive electrodynamic field by utilizing Proca's theory. Atomic spectroscopy is also examined, with the focus on the $21 \mathrm{~cm}$ radiation due to the hyperfine structure of hydrogen. The modifications to Snell's Law, the Fresnel formulas, and the $21 \mathrm{~cm}$ radiation are shown to reduce to the familiar expressions in the limit of zero photon mass. 


\section{ACKNOWLEDGEMENTS}

Thank you to my advisor Peter Leung, my thesis committee, PSU and the Department of Physics, my fellow classmates, coworkers, friends, family and loved ones for the patience and support. 
TABLE OF CONTENTS

ABSTRACT.

ACKNOWLEDGEMENTS .ll

TABLE OF CONTENTS III

TABLE OF FIGURES. V

1 INTRODUCTION 1

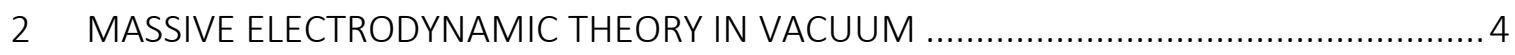

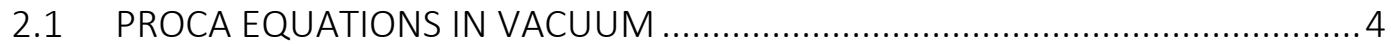

2.2 COVARIANT FORMULATION OF THE VACUUM FIELD EQUATIONS................ 6

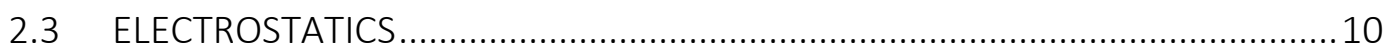

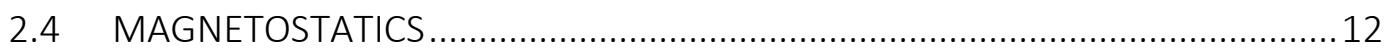

2.5 PLANE WAVE SOLUTIONS AND THE DISPERSION OF LIGHT .........................12

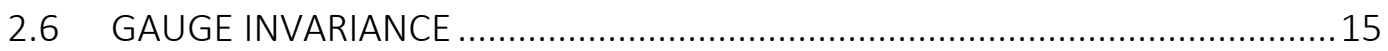

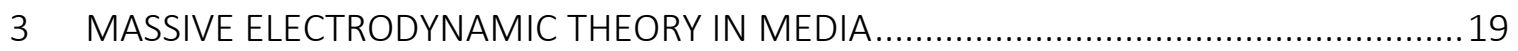

3.1 LAGRANGIAN AND THE PROCA EQUATIONS IN LIH MEDIA.........................19

3.2 WAVE EQUATIONS AND PLANE WAVE SOLUTIONS ...................................21

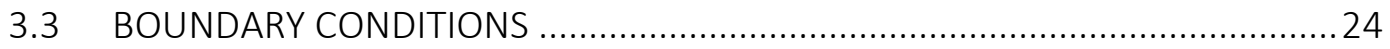

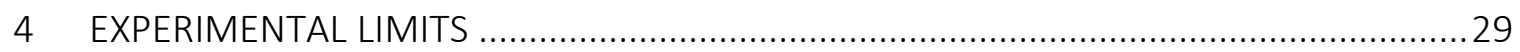

4.1 ELECTROSTATIC EXPERIMENTS: INVERSE SQUARE LAW ............................29

4.2 MAGNETOSTATIC EXPERIMENTS: VERIFICATION OF AMPERE'S LAW ..........31

4.3 ELECTRODYNAMIC EXPERIMENTS: DISPERSION OF LIGHT ............................ 32

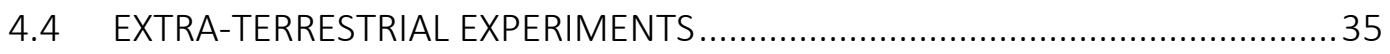

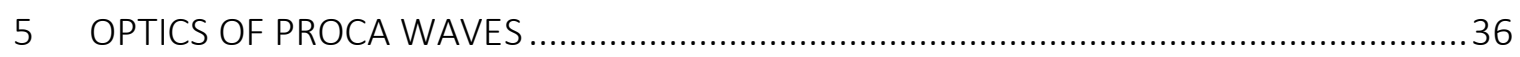

5.1 KINEMATICS: LAWS OF REFLECTION AND REFRACTION ...............................36 
5.2 DYNAMICS: TYPE I FRESNEL EQUATIONS …......................................... 40

5.3 DYNAMICS: TYPE II FRESNEL EQUATIONS .............................................. 43

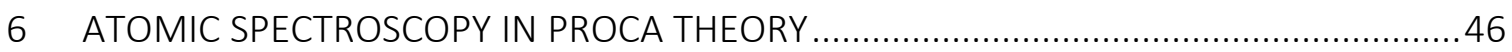

6.121 CM RADIATION IN THE MASSLESS LIMIT ...............................................46

6.2 GROUND STATE WAVE FUNCTION FOR THE YUKAWA POTENTIAL .............49

6.3 MAGNETIC DIPOLE FIELD FOR A MASSIVE PHOTON.................................... 51

6.4 REVISION TO THE 21 CM RADIATION IN PROCA THEORY .............................54

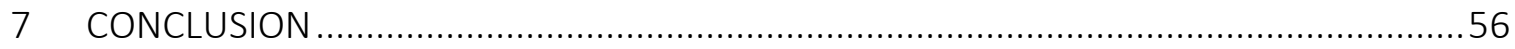

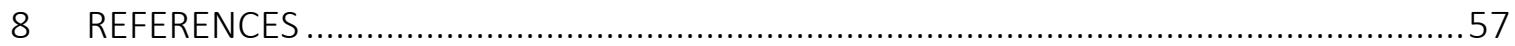




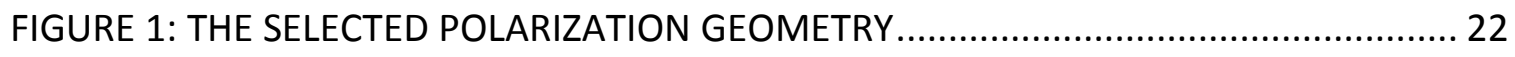

FIGURE 2: A SHALLOW 3-DIMENSIONAL CYLINDER ENCOMPASSING THE INTERFACE.... 25

FIGURE 3: A SHALLOW 2-DIMENSIONAL LOOP ENCOMPASSING THE INTERFACE........... 27

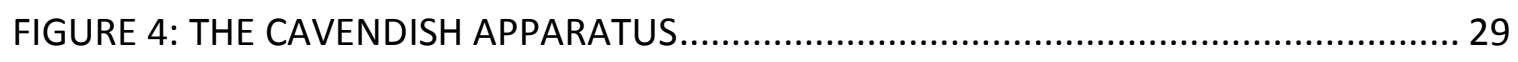

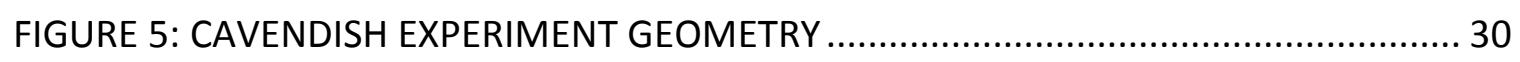

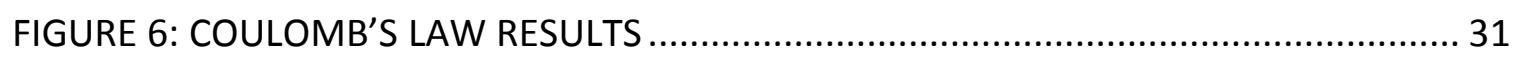

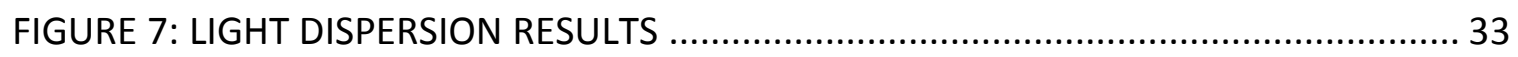

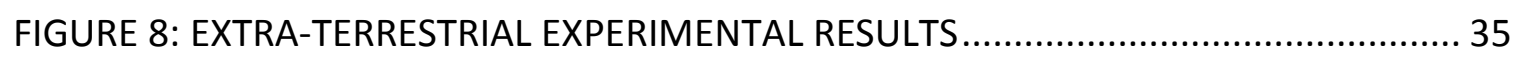

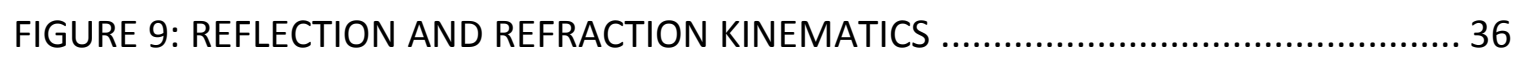

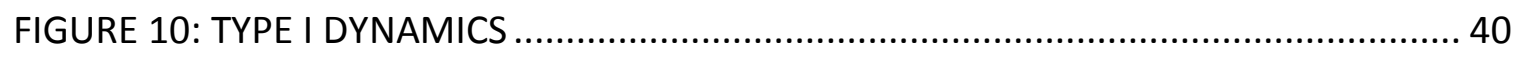

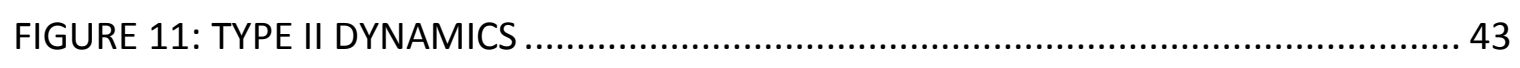


One of the main consequences of the widely accepted and utilized Maxwell equations is that the carrier of electromagnetic waves, the photon, possesses an identically zero rest mass. The features of Maxwell theory, and thus the assumption of a massless photon, have been thoroughly investigated: the inverse square law for electrostatic potentials, dispersionless propagation of electromagnetic waves in vacuum, gauge invariance, kinematics and dynamics in optics, and a multitude of other measureable phenomena. A nonzero photon mass $m_{\gamma}$ can be considered by modifications to Maxwell theory, the theoretical framework of which was provided by Proca. By considering a nonzero photon mass, all of the familiar expressions to describe these phenomena will need to be modified, likely by higher order terms of $m_{\gamma}$. The question of a nonzero photon mass is more than a purely academic one, as the guiding focus of these efforts through the years has been the confirmation of Maxwell electromagnetism. A theoretical study into these effects facilitates the measurement of increasingly stringent limits on the proposed photon mass $m_{\gamma}$, and thus further confirms a pivotal theory of modern physics.

The following thesis starts by examining some of the main features of Proca theory in vacuum (Chapter 2). This chapter summarizes previous theoretical accomplishments, beginning with Proca's formalism [1] [2] [3] [4] [5] and further investigating static fields [6], dynamic fields, and plane wave solutions [7], [8]. Some of the significant differences between Maxwell and Proca theory will be touched upon: the deviation from an inverse 
square law for electrostatics (Section 2.3), an additional longitudinal polarization and the dispersion of light (Section 2.5), and the loss of gauge invariance (Section 2.6).

Chapter 3 extends some of this work to linear, isotropic, and homogeneous media. The approach follows Jackson's for extending Maxwell theory to a magnetodielectric medium [9]. The plane wave solutions will be explicitly solved for the three polarizations of Proca theory, as well as the relevant boundary conditions. This has been attempted before for Proca theory for dielectric media [10], as well as full magnetodielectric media [11], though the approach here will differ slightly.

Chapter 4 will detail some of the experimental efforts to measure an upper limit for the photon mass using some of the previously established theoretical results. These efforts through the years have employed both static and dynamic techniques, and ranged from tabletop setups to the study of extraterrestrial data. To date, an accepted upper limit from these techniques is on the order of $m_{\gamma}<10^{-49} \mathrm{~g}$.

The primary original work herein will be Chapters 5 and 6 . In Chapter 5 we will examine modifications to the kinematics and dynamics in optics according to Proca theory. These will be calculable using the previously established theory for plane wave solutions in a medium. Necessary modifications to the Laws of Reflection and Refraction will be derived, as well as alterations to the Type I Fresnel formulas. As will be seen, significant mathematical difficulties arise when solving the Fresnel formulas for the Type II and Type 
III polarizations, though in principle these are possible to work out, as shown in [10] and [11].

Chapter 6 will investigate modifications to atomic spectroscopy in Proca theory, specifically the hyperfine splitting of Hydrogen atoms and the resulting $21 \mathrm{~cm}$ radiation. The theory mirrors the Maxwell case except for two key features: the magnetic dipole field [12] and the ground state wavefunction for Hydrogen [13]. The necessary modifications according to Proca theory will be examined, and the first order correction to the $21 \mathrm{~cm}$ spectral line will be derived.

These revisions to kinematic optics, dynamic optics, and spectroscopy all provide measureable means of improving the photon mass upper limit. The macroscopic nature of optics limits its utility in improving these measurements. However, the microscopic nature of the $21 \mathrm{~cm}$ radiation result may provide a more promising application for these ever-improving measurements of a fundamental cornerstone of modern physics. 


\subsection{PROCA EQUATIONS IN VACUUM}

The Proca equations are the simplest Lorentz-invariant revision to the Maxwell equations that incorporate a non-zero photon mass $m_{\gamma}$, or more usefully expressed as the inverse Compton wavelength $\mu_{\gamma}$,

$$
\mu_{r}=\frac{1}{\lambda_{\gamma}}=\frac{c m_{\gamma}}{\hbar}
$$

Before delving into the covariant approach that prompted Proca to adjust the Maxwell Lagrangian, it will be instructive to see the Proca Equations in familiar vector notation and see the immediate consequences of a non-zero photon mass. In Gaussian units, the equations are as follows:

$$
\begin{aligned}
& \boldsymbol{\nabla} \cdot \mathbf{E}=4 \pi \rho-\mu_{\gamma}^{2} \Phi \\
& \boldsymbol{\nabla} \cdot \mathbf{B}=0 \\
& \boldsymbol{\nabla} \times \mathbf{E}+\frac{1}{c} \frac{\partial \mathbf{B}}{\partial t}=0 \\
& \boldsymbol{\nabla} \times \mathbf{B}-\frac{1}{c} \frac{\partial \mathbf{E}}{\partial t}=\frac{4 \pi}{c} \mathbf{J}-\mu_{\gamma}^{2} \mathbf{A}
\end{aligned}
$$

The most notable modification is the direct inclusion of the potentials in the equations. In Maxwell electromagnetism, the potentials are viewed as a convenient abstraction, while the fields possess real and quantifiable characteristics through the Lorentz force 
equation. However, in Proca electromagnetism the potentials take on some of the characteristics of the fundamental electromagnetic quantity.

The sourceless equations (2.3) and (2.4) are unchanged by the photon mass, and as in Maxwell theory lead to the definition of the potentials,

$$
\begin{gathered}
\mathbf{B}=\boldsymbol{\nabla} \times \mathbf{A} \\
\mathbf{E}=-\nabla \Phi-\frac{1}{c} \frac{\partial \mathbf{A}}{\partial t}
\end{gathered}
$$

Local conservation of charge is still assumed, expressed as the equation of continuity,

$$
\boldsymbol{\nabla} \cdot \mathbf{J}+\frac{\partial \rho}{\partial t}=0
$$

Upon taking the divergence of (2.5) and substituting (2.2) we find,

$$
\mu_{\gamma}^{2}\left(\boldsymbol{\nabla} \cdot \mathbf{A}+\frac{1}{c} \frac{\partial \Phi}{\partial t}\right)=\frac{4 \pi}{c}\left(\boldsymbol{\nabla} \cdot \mathbf{J}+\frac{\partial \rho}{\partial t}\right)
$$

Hence, if local charge conservation (2.8) is assumed, a non-zero photon mass forces the Lorentz gauge,

$$
\boldsymbol{\nabla} \cdot \mathbf{A}+\frac{1}{c} \frac{\partial \Phi}{\partial t}=0
$$

Proca theory can be developed as a consistent theory satisfying charge conservation, but with the cost of abandoning gauge invariance. It is this feature of Proca theory that prompted many through the years to assume on a fundamental basis the photon is 
massless so that gauge invariance can be upheld. In Section 2.6 the question of gauge invariance and its apparent loss in Proca theory will be further investigated.

\subsection{COVARIANT FORMULATION OF THE VACUUM FIELD EQUATIONS}

Between the years of 1936 and 1938, Alexandru Proca developed a consistent relativistic field theory for spin-1 particles [1] [2] [3] [4] [5]. The theory was Lorentz-invariant and described massive or massless spin-1 particles. Though the theory is an appropriate modification to Maxwell's theory to describe massive photons, this fundamental question did not seem to be the primary motivation. The theory has broad applications, for both inter-nucleon interactions as well as electromagnetism. At the time, Yukawa was developing a meson theory to describe inter-nucleon forces [6]. He proposed a scalar field which predicted the existence of massive spin-0 pions. Spin-1 mesons require the full vector-field theory of Proca.

In the following, conventional covariant notation will be employed. Of particular use will be the Einstein repeated index sum convention. In this notation, a contravariant 4position vector will be expressed as,

$$
x^{\alpha}=(c t, x, y, z)
$$

Only the flat space-time of special relativity will be considered. The metric tensor to describe this will be the usual Minkowski metric $g_{\alpha \beta}$, 


$$
g_{\alpha \beta}=g^{\alpha \beta}=\left[\begin{array}{rrrr}
1 & 0 & 0 & 0 \\
0 & -1 & 0 & 0 \\
0 & 0 & -1 & 0 \\
0 & 0 & 0 & -1
\end{array}\right]
$$

A covariant vector can be obtained by contracting a contravariant vector with the metric tensor.

$$
x_{\alpha}=g_{\alpha \beta} x^{\beta}=(c t,-x,-y,-z)
$$

The 4-potential will be composed of the electric scalar potential $\Phi$ and the magnetic vector potential A,

$$
A^{\alpha}=\left(\Phi, A_{x}, A_{y}, A_{z}\right)
$$

The 4-current will similarly be expressed as a combination of the scalar charge density $\rho$ and the vector current density $\mathbf{J}$,

$$
J^{\alpha}=\left(c \rho, J_{x}, J_{y}, J_{z}\right)
$$

Contravariant and covariant derivatives will be defined as,

$$
\partial^{\alpha}=\frac{\partial}{\partial x_{\alpha}} \quad \partial_{\alpha}=\frac{\partial}{\partial x^{\alpha}} \quad \partial_{\alpha} \partial^{\alpha}=\frac{1 \partial^{2}}{c^{2} \partial t^{2}}-\nabla^{2}
$$


In this notation, the continuity of charge (2.8) and Lorentz condition (2.10) take on a particularly convenient form:

$$
\begin{gathered}
\partial^{\alpha} J_{\alpha}=0 \\
\partial^{\alpha} A_{\alpha}=0
\end{gathered}
$$

It is useful to construct a $4 \times 4$ tensor out of the components of the electric field $\mathbf{E}$ and the magnetic induction B (or equivalently the derivatives of the 4-potential, through equations (2.6) \& (2.7)), called the Field-Strength Tensor $F^{\alpha \beta}$,

$$
F^{\alpha \beta}=\partial^{\alpha} A^{\beta}-\partial^{\beta} A^{\alpha}=\left[\begin{array}{rrrr}
0 & -E_{x} & -E_{y} & -E_{z} \\
E_{x} & 0 & -B_{z} & B_{y} \\
E_{y} & B_{z} & 0 & -B_{x} \\
E_{z} & -B_{y} & B_{x} & 0
\end{array}\right]
$$

The covariant field-strength tensor $F_{\alpha \beta}$ can be found through contraction with the metric tensor,

$$
F_{\alpha \beta}=g_{\alpha \gamma} F^{\gamma \delta} g_{\delta \beta}=\partial_{\alpha} A_{\beta}-\partial_{\beta} A_{\alpha}=\left[\begin{array}{rrrr}
0 & E_{x} & E_{y} & E_{z} \\
-E_{x} & 0 & -B_{z} & B_{y} \\
-E_{y} & B_{z} & 0 & -B_{x} \\
-E_{z} & -B_{y} & B_{x} & 0
\end{array}\right]
$$

Also useful in the covariant approach is the Dual Field-Strength tensor, 


$$
\mathcal{F}^{\alpha \beta}=\frac{1}{2} \epsilon^{\alpha \beta \gamma \delta} F_{\gamma \delta}=\left[\begin{array}{rrrr}
0 & -B_{x} & -B_{y} & -B_{z} \\
B_{x} & 0 & -E_{z} & -E_{y} \\
B_{y} & -E_{z} & 0 & E_{x} \\
B_{z} & E_{y} & -E_{x} & 0
\end{array}\right]
$$

The Levi-Civita symbol $\epsilon^{\alpha \beta \gamma \delta}$ is employed here, also known as the permutation tensor or the complete antisymmetric tensor. In general it can be extended to any tensor rank,

$$
\epsilon^{\alpha \beta \gamma \delta}=\left\{\begin{array}{c}
+1 \text { for } \alpha, \beta, \gamma, \delta=1,2,3,4 \text { or any even permutation } \\
-1 \text { for any odd permuation } \\
0 \text { for any repeated index }
\end{array}\right.
$$

Maxwell electromagnetism can be framed in a covariant approach by beginning with the electromagnetic Lagrangian $\mathcal{L}$ including both field and source terms.

$$
\mathcal{L}=-\frac{1}{16 \pi} F_{\alpha \beta} F^{\alpha \beta}-\frac{1}{c} J_{\alpha} A^{\alpha}
$$

The Proca Lagrangian is a simple revision to this, with the inclusion of a mass term. It is the simplest mass term that can be added and still maintain Lorentz invariance.

$$
\mathcal{L}=-\frac{1}{16 \pi} F_{\alpha \beta} F^{\alpha \beta}-\frac{1}{c} J_{\alpha} A^{\alpha}+\frac{\mu_{\gamma}^{2}}{8 \pi} A_{\alpha} A^{\alpha}
$$

The equation of motion can be obtained through the usual Lagrangian approach for continuous fields. 


$$
\partial^{\beta} \frac{\partial \mathcal{L}}{\partial\left(\partial^{\beta} A^{\alpha}\right)}=\frac{\partial \mathcal{L}}{\partial A^{\alpha}} \quad \rightarrow \quad \partial^{\beta} F_{\beta \alpha}+\mu_{\gamma}^{2} A_{\alpha}=\frac{4 \pi}{c} J_{\alpha}
$$

Equation (2.23) contains all the information in covariant notation from the Proca source equations, (2.2) and (2.5). The sourceless equations are contained implicitly in the definition of the field-strength tensor $F^{\alpha \beta}$, just as in the Maxwell case. The notation is simpler if the dual field-strength tensor is employed,

$$
\partial_{\alpha} \mathcal{F}^{\alpha \beta}=0
$$

Equation (2.24) contains all the sourceless information, or equations (2.3) and (2.4). Together, equations (2.23) and (2.24) succinctly express the Proca equations in covariant notation.

\subsection{ELECTROSTATICS}

In the electrostatic case, the electric field E from Equation (2.7), as in Maxwell theory reduces to the static form,

$$
\mathbf{E}=-\nabla \Phi
$$

The modified Coulomb's Law (2.2) can be expressed as a screened Poisson Equation,

$$
\left[\nabla^{2}-\mu_{\gamma}^{2}\right] \Phi=-4 \pi \rho
$$


Equation (2.26) is similar in form to the Maxwell electrostatic case, but with the addition of the photon mass term. This form appears in other contexts, and is named as such for its use in modeling charge screening due to the presence of other charge carriers, such as for modeling plasmas and semiconductors. The solution to the inhomogeneous Equation (2.26) is well documented, and solvable via the method of Fourier transform and Green function, resulting in the general form,

$$
\Phi(\mathbf{r})=\int \frac{\rho\left(\mathbf{r}^{\prime}\right) e^{-\mu_{\gamma}\left|\mathbf{r}-\mathbf{r}^{\prime}\right|}}{\left|\mathbf{r}-\mathbf{r}^{\prime}\right|} d^{3} r^{\prime}
$$

Or in the case of a point charge at the origin, $\rho\left(\mathbf{r}^{\prime}\right)=q \delta\left(r^{\prime}\right)$ the potential takes the form,

$$
\Phi(r)=\frac{q}{r} e^{-\mu_{\gamma} r}
$$

A potential of the form (2.28) is referred to as the Screened Poisson Potential or the Yukawa Potential. The electric field for this potential is given by,

$$
\mathbf{E}(\mathbf{r})=q\left(\frac{1}{r^{2}}+\frac{\mu_{\gamma}}{r}\right) e^{-\mu_{\gamma} r}
$$

The inverse square law for electrostatics has been well studied and measured. Thus, (2.29) provides a direct means for measuring an upper bound to the photon mass, as will be seen in Section 4.1. 


\subsection{MAGNETOSTATICS}

The magnetostatic case is similar in approach and results to the electrostatic one. With $\partial \mathbf{B} / \partial t=0$, in general $\Phi(\mathbf{r})$ will again follow a Yukawa-type potential (2.27). Replacing the potentials in Ampère's Law (2.5), and simplifying the form using the Lorentz condition (2.10) (which reduces to the Coulomb gauge in the static case: $\boldsymbol{\nabla} \cdot \mathbf{A}=0$ ) we get a vectorial screened Poisson equation for $\mathbf{A}$,

$$
\left[\nabla^{2}-\mu_{\gamma}^{2}\right] \mathbf{A}=-\frac{4 \pi}{c} \mathbf{J}
$$

Equation (3.32) admits Yukawa form solutions for each component,

$$
\mathbf{A}(\mathbf{r})=\int \frac{\mathbf{J}\left(\mathbf{r}^{\prime}\right) e^{-\mu_{\gamma}|\mathbf{r}-\mathbf{r} \prime|}}{c\left|\mathbf{r}-\mathbf{r}^{\prime}\right|} d^{3} r^{\prime}
$$

\subsection{PLANE WAVE SOLUTIONS AND THE DISPERSION OF LIGHT}

We now turn to full electrodynamics. Inputting the definitions of the potentials (2.6) \& (2.7) into the source equations (2.2) \& (2.5) as well as the inclusion of the now required Lorentz condition (2.10), yields the wave equations,

$$
\begin{aligned}
& {\left[\nabla^{2}-\frac{1}{c^{2}} \frac{\partial^{2}}{\partial t^{2}}-\mu_{\gamma}^{2}\right] \Phi=-4 \pi \rho} \\
& {\left[\nabla^{2}-\frac{1}{c^{2}} \frac{\partial^{2}}{\partial t^{2}}-\mu_{\gamma}^{2}\right] \mathbf{A}=-\frac{4 \pi}{c} \mathbf{J}}
\end{aligned}
$$


For Proca waves propagating in free space $(\rho=0, \mathbf{J}=\mathbf{0})$, each component equation is the Klein-Gordon equation, and admits plane wave solutions of the form,

$$
\begin{gathered}
\Phi(\mathbf{r}, t)=\Phi e^{i(\mathbf{k} \cdot \mathbf{r}-\omega t)} \\
\mathbf{A}(\mathbf{r}, t)=A \hat{\mathbf{a}} e^{i(\mathbf{k} \cdot \mathbf{r}-\omega t)}
\end{gathered}
$$

Substitution of these forms into the wave equations yields the dispersion relation,

$$
k^{2}=\frac{\omega^{2}}{c^{2}}-\mu_{\gamma}^{2}
$$

Thus in Proca theory, light is dispersive. As with all dispersive waves, the speed of the wave will now be best described by the group velocity and phase velocity,

$$
\begin{aligned}
& v_{p}=\frac{\omega}{k}=c\left(1-\frac{\mu_{\gamma}^{2} c^{2}}{\omega^{2}}\right)^{-1 / 2} \approx c\left(1+\frac{\mu_{\gamma}^{2} c^{2}}{2 \omega^{2}}\right) \\
& v_{g}=\frac{d \omega}{d k}=c\left(1-\frac{\mu_{\gamma}^{2} c^{2}}{\omega^{2}}\right)^{1 / 2} \approx c\left(1-\frac{\mu_{\gamma}^{2} c^{2}}{2 \omega^{2}}\right)
\end{aligned}
$$

As can be seen from (2.37) and (2.38), the phase and group velocity both converge to $c$ if the photon mass is taken to zero. The constancy of the speed of light is taken as an axiom in special relativity, and so this new feature of Proca theory would seem to be at odds with Einstein's theory. This would present a consistency problem, as the special theory of relativity guided the Lorentz invariant mass term that is the basis of Proca theory (2.22). 
However, relativity can still be perfectly consistent with Proca theory if the axiomatic constant speed $c$ in relativity is viewed as a limiting speed of light when $\omega \rightarrow \infty$. Indeed this is the approach taken in equations (2.37) and (2.38), with $c$ being held constant, and the group and phase velocity of light varying with frequency.

Proca theory will in general admit three polarizations of light. To see this clearly, the following vector identity can be employed: $\boldsymbol{\nabla} \times(\boldsymbol{\nabla} \times \mathbf{A})=\boldsymbol{\nabla}(\boldsymbol{\nabla} \cdot \mathbf{A})-\nabla^{2} \mathbf{A}$. Taking the derivatives of the plane wave solution form in the sourceless equation (2.33), we find,

$$
\mathbf{k} \times(\mathbf{k} \times \mathbf{A})-\mathbf{k}(\mathbf{k} \cdot \mathbf{A})+\frac{\omega^{2}}{c^{2}} \mathbf{A}-\mu_{\gamma}^{2} \mathbf{A}=0
$$

The two classes of polarizations can be readily seen from (2.39). In general there will be two transverse polarizations with $\mathbf{k} \cdot \mathbf{A}=0$, and one longitudinal polarization with $\mathbf{k} \times$ $\mathbf{A}=\mathbf{0}$. In free space, all three polarizations will obey the same dispersion relation (2.36). The transverse polarizations, here referred to as Type I and Type II, have direct analogs to the TE and TM polarizations of Maxwell theory. The longitudinal polarization, Type III, is a wholly new feature of Proca theory. In the massless limit, the longitudinal polarization disappears. To see this, we need to turn to the fields, as they are the fundamental electromagnetic quantities in Maxwell theory. From the plane wave form (2.35), the fields for all polarizations will be given by, 


$$
\mathbf{E}=i\left(\frac{\omega}{c} A \hat{\mathbf{a}}-\Phi \mathbf{k}\right) e^{i(\mathbf{k} \cdot \mathbf{r}-\omega t)}
$$

$$
\mathbf{B}=i A(\mathbf{k} \times \hat{\mathbf{a}}) e^{i(\mathbf{k} \cdot \mathbf{r}-\omega t)}
$$

The Type III polarization will simplify to,

$$
\begin{gathered}
\mathbf{E}=i\left(\frac{\omega}{k c} A-\Phi\right) \mathbf{k} e^{i(\mathbf{k} \cdot \mathbf{r}-\omega t)} \\
\mathbf{B}=0
\end{gathered}
$$

Substituting (2.42) into Proca's equation (2.2), we arrive at the dispersion relation,

$$
\frac{k \omega}{c} A-\left(k^{2}+\mu_{\gamma}^{2}\right) \Phi=0
$$

In the massless limit $\mu_{\gamma} \rightarrow 0$, the dispersion relation (2.36) will approach the familiar Maxwell form $k=\omega / c$. Therefore, Equation (2.44) implies $A=\Phi$ and Equation (2.42) will then imply $\mathbf{E}=\mathbf{0}$. Hence, in Maxwell theory, Type III longitudinal waves only satisfy all the necessary equations if $\mathbf{E}=\mathbf{B}=\mathbf{0}$, or equivalently, if they do not exist.

\subsection{GAUGE INVARIANCE}

As discussed in Section 2.1, the loss of gauge invariance is one of the key differences between Maxwell and Proca theory. Without abandoning charge conservation, this is an 
inevitable feature of Proca theory, and one of the key fundamental reasons that the photon mass is assumed to be zero in many contexts. As explained in Goldhaber et al [8], this is a consequence of the minimal dynamics being considered. The Proca Lagrangian mass term,

$$
\mathcal{L}_{\mu_{\gamma}}=\frac{\mu_{\gamma}^{2}}{8 \pi} A_{\alpha} A^{\alpha}
$$

admits no further dynamics, i.e. is formulated in a Lorentz invariant way out of the existing electromagnetic potentials. However, if additional dynamics are taken into account in the form of additional scalar fields, then both gauge invariance and a finite photon mass can be shown to not be mutually exclusive.

The first such attempt was by Stueckelberg [14]. He proposed a new scalar field $\Lambda$ with a fixed magnitude carrying electric charge $q_{\Lambda}$. The proposed photon mass Lagrangian term was,

$$
\mathcal{L}_{\mu_{\gamma}}=\frac{1}{8 \pi}\left[\left|-\partial_{t} \Lambda+\frac{i q_{\Lambda}}{\hbar} \Lambda \Phi\right|^{2}-\left|\nabla \Lambda-\frac{i q_{\Lambda}}{\hbar} \Lambda \mathbf{A}\right|^{2}\right]
$$

The Proca term can be recovered from this form by restricting the gauge for this new field and taking $\Lambda$ to be constant. In particular, we can define the photon mass in terms of this new field, 


$$
\mu_{\gamma} \equiv \frac{q_{\Lambda}}{\hbar} \Lambda
$$

It can be seen that this constant value for this new field will result in the recovery of the Proca term (2.45) from the general Stueckelberg term (2.46). In this case, gauge invariance is only lost because a specific choice was made for the field $\Lambda$. Without such a step, both gauge invariance and a photon mass are possible, though at the cost of additional complexity by considering another scalar field $\Lambda$ interacting with the photon vector field $A^{\alpha}$.

These ideas were furthered in the 1960's with the development of the Higg's mechanism. A number of contributors (for full citations see [8]) developed the idea of a dynamic massgenerating field. A representation of this in the current context is given by an additional term in Stueckelberg's expression (2.46),

$$
\mathcal{L}_{\mu_{\gamma}}=\frac{1}{8 \pi}\left[\left|-\partial_{t} \Lambda+\frac{i q_{\Lambda}}{\hbar} \Lambda \Phi\right|^{2}-\left|\nabla \Lambda-\frac{i q_{\Lambda}}{\hbar} \Lambda \mathbf{A}\right|^{2}\right]-\frac{\lambda}{16 \pi}\left(|\Lambda|^{2}-\langle|\Lambda|\rangle^{2}\right)
$$

The term $\langle|\Lambda|\rangle$ is the vacuum expectation value, and encapsulates the concept of a nonzero magnitude for the field while in the ground state. The advantage of this approach over that of Stueckelberg's is that under certain circumstances, such as high temperature or a strong electromagnetic field $A^{\alpha}$, the scalar field $\Lambda$ can be forced to vanish. Thus the photon mass under certain circumstances can be identically zero as commonly assumed, and in other cases take on a non-zero mass. And similar to Stueckelberg's expression, by 
choosing a static constant value for $\Lambda$ in equation (2.48), Proca's term (2.45) can be recovered.

Throughout the following work, the simplest Proca term will be employed, though at the cost of the loss of gauge invariance. 
The theory of the preceding section can be generalized to linear, isotropic, and homogeneous media in a fairly straightforward way. We will restrict our investigation to this simple subset of media to enable the consideration of some general consequences of a massive photon in macroscopic media.

\subsection{LAGRANGIAN AND THE PROCA EQUATIONS IN LIH MEDIA}

The Proca equations (2.2) - (2.5) can be extended to macroscopic media analogously to the Maxwell equations [9], [15]. Assuming only linear, isotropic, and homogeneous media (lih), the electric field $\mathbf{E}$ and the magnetic induction $\mathbf{B}$ are linearly proportional to the electric displacement $\mathrm{D}$ and magnetic field $\mathrm{H}$,

$$
\begin{aligned}
& \mathbf{D}=\varepsilon \mathbf{E} \\
& \mathbf{B}=\mu \mathbf{H}
\end{aligned}
$$

Following a manifestly covariant approach, a new field-strength tensor can be constructed of the macroscopic fields $\mathrm{D}$ and $\mathrm{H}$,

$$
G^{\alpha \beta}=\left[\begin{array}{rrrr}
0 & -D_{x} & -D_{y} & -D_{z} \\
D_{x} & 0 & -H_{z} & H_{y} \\
D_{y} & H_{z} & 0 & -H_{x} \\
D_{z} & -H_{y} & H_{x} & 0
\end{array}\right]
$$


The only change to the Lagrangian will be to the field term, which will be adjusted with the inclusion of the new field-strength tensor (3.3). The source term will remain unchanged in form, with the understanding that only free unbound charges are to be considered. Notably, there is no modification necessary to the mass term, as the simplest covariant form still only includes the potential, and is unaffected by the consideration of a medium.

$$
\mathcal{L}=-\frac{1}{16 \pi} F_{\alpha \beta} G^{\alpha \beta}-\frac{1}{c} J_{\alpha} A^{\alpha}+\frac{\mu_{\gamma}^{2}}{8 \pi} A_{\alpha} A^{\alpha}
$$

Similar to the case in vacuum, this will lead to the field equations,

$$
\begin{aligned}
& \partial^{\beta} G_{\beta \alpha}+\mu_{\gamma}^{2} A_{\alpha}=\frac{4 \pi}{c} J_{\alpha} \\
& \partial_{\alpha} \mathcal{F}^{\alpha \beta}=0
\end{aligned}
$$

Expressed in vector notation, equations (3.5) and (3.6) lead to the macroscopic Proca equations,

$$
\begin{aligned}
& \boldsymbol{\nabla} \cdot \mathbf{D}=4 \pi \rho-\mu_{\gamma}^{2} \Phi \\
& \boldsymbol{\nabla} \cdot \mathbf{B}=0
\end{aligned}
$$




$$
\begin{aligned}
& \boldsymbol{\nabla} \times \mathbf{E}+\frac{1}{c} \frac{\partial \mathbf{B}}{\partial t}=0 \\
& \boldsymbol{\nabla} \times \mathbf{H}-\frac{1}{c} \frac{\partial \mathbf{D}}{\partial t}=\frac{4 \pi}{c} \mathbf{J}-\mu_{\gamma}^{2} \mathbf{A}
\end{aligned}
$$

\subsection{WAVE EQUATIONS AND PLANE WAVE SOLUTIONS}

The wave equations are found through the same approach as the vacuum case. However, with the required Lorentz gauge and non-unity $\varepsilon$ and $\mu$, the equations will no longer take on a wave equation form. In Maxwell theory, the reduced Lorentz gauge is usually chosen in a medium to recover a wave equation,

$$
\boldsymbol{\nabla} \cdot \mathbf{A}+\frac{\varepsilon \mu}{c} \frac{\partial \Phi}{\partial t}=0
$$

However, this is not possible in Proca theory, as the gauge condition (2.10) is still enforced due to charge conservation. This leads to the more complicated forms,

$$
\begin{aligned}
& {\left[\nabla^{2}-\frac{1}{c^{2}} \frac{\partial^{2}}{\partial t^{2}}-\frac{\mu_{\gamma}^{2}}{\varepsilon}\right] \Phi=-\frac{4 \pi \rho}{\varepsilon}} \\
& {\left[\nabla^{2}-\frac{\varepsilon \mu}{c^{2}} \frac{\partial^{2}}{\partial t^{2}}-\mu \mu_{\gamma}^{2}\right] \mathbf{A}+(\varepsilon \mu-1) \boldsymbol{\nabla}(\boldsymbol{\nabla} \cdot \mathbf{A})=-\frac{4 \pi \mu}{c} \mathbf{J}}
\end{aligned}
$$


As in the vacuum case of Section 2.5, these equations allow the Type I and Type II transverse polarizations with $\mathbf{k} \cdot \mathbf{A}=0$, and the Type III longitudinal polarization with $\mathbf{k} \times$ $\mathbf{A}=\mathbf{0}$. However, unlike the vacuum case, the transverse and longitudinal polarizations will obey different dispersion relations due to the asymmetric form of the wave equations. Without loss of generality, in the following it will be assumed that $\mathbf{k} \in(x, z)$ plane (see Figure 1). It will be useful for subsequent chapters if the angle $\theta$ is employed.

Figure 1: The selected polarization geometry

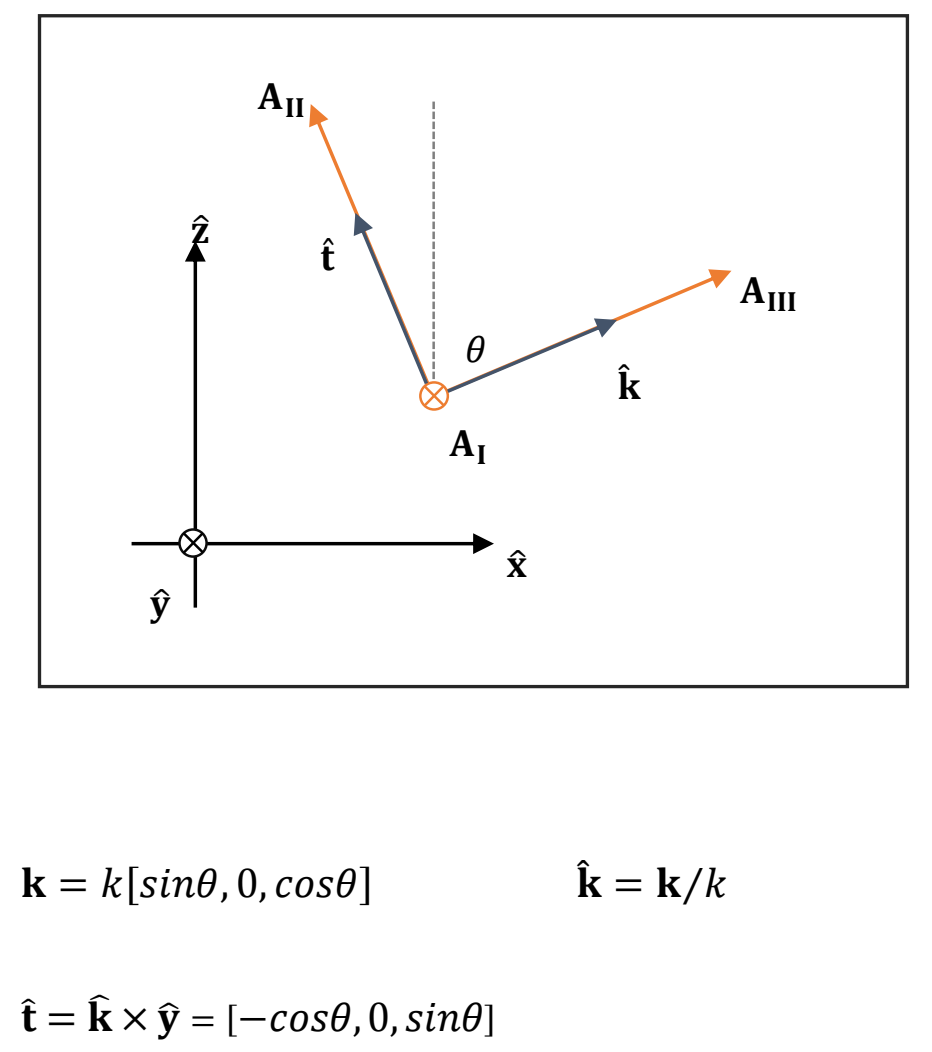

For this selected geometry, the dispersion relations, potentials, and fields for the three polarization types can be summarized as follows: 
Type I Transverse Mode:

Dispersion Relation:

$$
k^{2}=\frac{\varepsilon \mu \omega^{2}}{c^{2}}-\mu \mu_{\gamma}^{2}
$$

$$
\text { Potentials: } \quad \begin{aligned}
\mathbf{A} & =A \hat{\mathbf{y}} e^{i(\mathbf{k} \cdot \mathbf{r}-\omega t)} \\
\Phi & =0 \\
\text { Fields: } \quad & \mathbf{E}=\frac{i \omega}{c} A \hat{\mathbf{y}} e^{i(\mathbf{k} \cdot \mathbf{r}-\omega t)} \\
& \mathbf{B}=i k A \hat{\mathbf{t}} e^{i(\mathbf{k} \cdot \mathbf{r}-\omega t)}
\end{aligned}
$$

Type II Transverse Mode:

Dispersion Relation:

$$
k^{2}=\frac{\varepsilon \mu \omega^{2}}{c^{2}}-\mu \mu_{\gamma}^{2}
$$

$$
\text { Potentials: } \quad \begin{aligned}
\mathbf{A} & =A \hat{\mathbf{t}} e^{i(\mathbf{k} \cdot \mathbf{r}-\omega t)} \\
\Phi & =0 \\
\text { Fields: } \quad & \mathbf{E}=\frac{i \omega}{c} A \hat{\mathbf{t}} e^{i(\mathbf{k} \cdot \mathbf{r}-\omega t)} \\
\mathbf{B} & =-i k A \hat{\mathbf{y}} e^{i(\mathbf{k} \cdot \mathbf{r}-\omega t)}
\end{aligned}
$$

Type III Longitudinal Mode:

Dispersion Relation:

$$
k^{2}=\frac{\omega^{2}}{c^{2}}-\frac{\mu_{\gamma}^{2}}{\varepsilon}
$$

Potentials:

$$
\begin{aligned}
& \mathbf{A}=A \hat{\mathbf{k}} e^{i(\mathbf{k} \cdot \mathbf{r}-\omega t)} \\
& \Phi=\frac{c k}{\omega} A e^{i(\mathbf{k} \cdot \mathbf{r}-\omega t)}
\end{aligned}
$$




$$
\text { Fields: } \quad \begin{aligned}
\mathbf{E} & =\frac{i c \mu_{\gamma}^{2}}{\varepsilon \omega} A \hat{\mathbf{k}} e^{i(\mathbf{k} \cdot \mathbf{r}-\omega t)} \\
\mathbf{B} & =\mathbf{0}
\end{aligned}
$$

\subsection{BOUNDARY CONDITIONS}

The boundary conditions for the macroscopic Proca fields given by equations (3.7) (3.10), as will be shown, are identical to the Maxwell conditions. There will also be some necessary boundary conditions for the potentials, as the potentials take on the role of the fundamental quantity in Proca theory, as well as being of fixed gauge. According to Kroll [16], as seen through a Green function representation of $\mathbf{A}$ and $\Phi$, all potential components must be continuous across a boundary.

$$
\begin{aligned}
& \mathbf{A}_{\|}=\mathbf{A}_{\|}^{\prime} \\
& \mathbf{A}_{\perp}=\mathbf{A}_{\perp}^{\prime} \\
& \Phi=\Phi^{\prime}
\end{aligned}
$$


Figure 2: a shallow 3-dimensional cylinder encompassing the interface

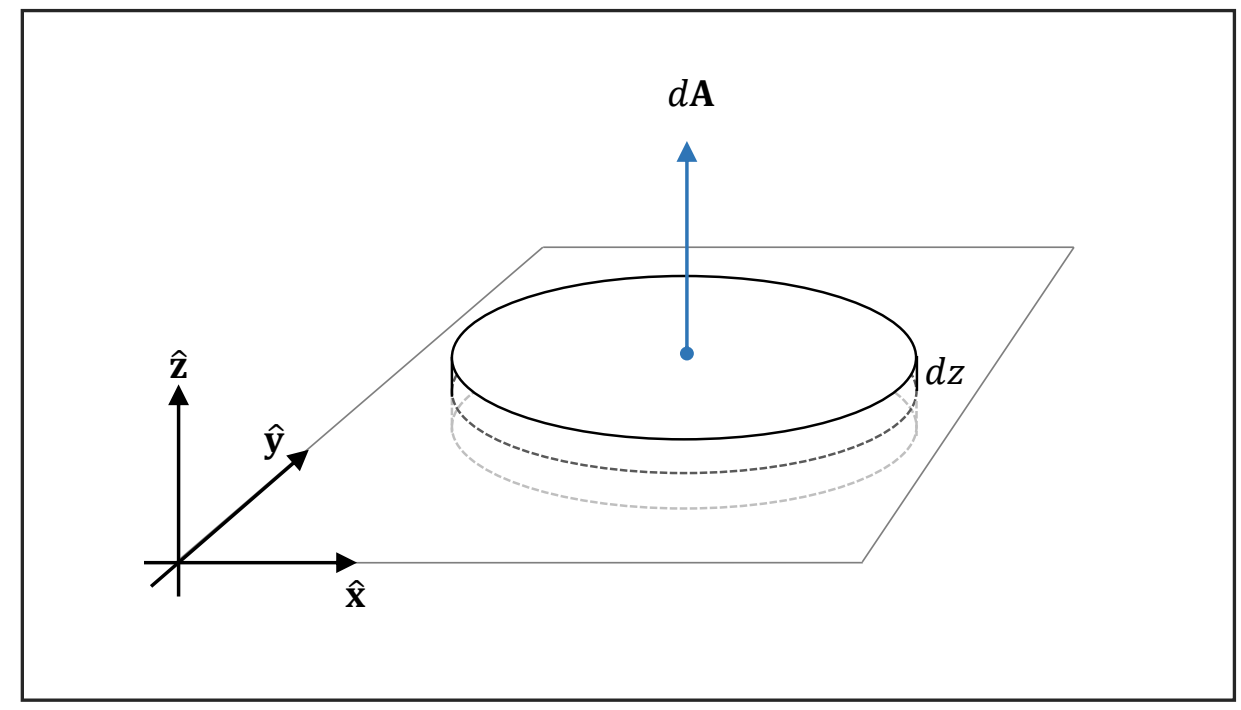

The field boundary conditions can be worked out from the Proca equations in an analogous method to Jackson [9]. Equation (3.7) leads to the boundary condition for the electric displacement $\mathbf{D}$. This can be seen by integrating the differential form over a shallow cylinder encompassing the boundary (Figure 2). In general we allow the interface to have a surface charge $\rho=\sigma \delta(z)$. The integral of this form is given by,

$$
\int \boldsymbol{\nabla} \cdot \mathbf{D} d V=4 \pi \int \rho d V-\mu_{\gamma}^{2} \int \Phi d V
$$

Taking the limit $d z \rightarrow 0$, the $\Phi$ term will approach 0 , as it is taken to be continuous and is integrated over a zero volume. By using the divergence theorem, we can re-write Equation (3.28), 


$$
\int\left(\mathbf{D}^{\prime}-\mathbf{D}\right) \cdot \hat{\mathbf{z}} d A=4 \pi \int \sigma d A
$$

From which the usual boundary condition can be extracted,

$$
\mathbf{D}_{\perp}^{\prime}-\mathbf{D}_{\perp}=4 \pi \sigma
$$

Thus if there is a surface charge, the discontinuity of the normal component of the electric displacement $\mathbf{D}$ is given by that charge. If there is no surface charge present, then the normal component of $\mathrm{D}$ is continuous.

The condition for $\mathbf{B}$ is similar and follows the same approach as for D starting from Equation (3.8). Employing the same geometry and divergence theorem, as there are no magnetic sources,

$$
\mathbf{B}_{\perp}^{\prime}-\mathbf{B}_{\perp}=0
$$

The conditions for the curl equations will require a different geometry, a square loop encompassing the interface (Figure 3): 
Figure 3: a shallow 2-dimensional loop encompassing the interface

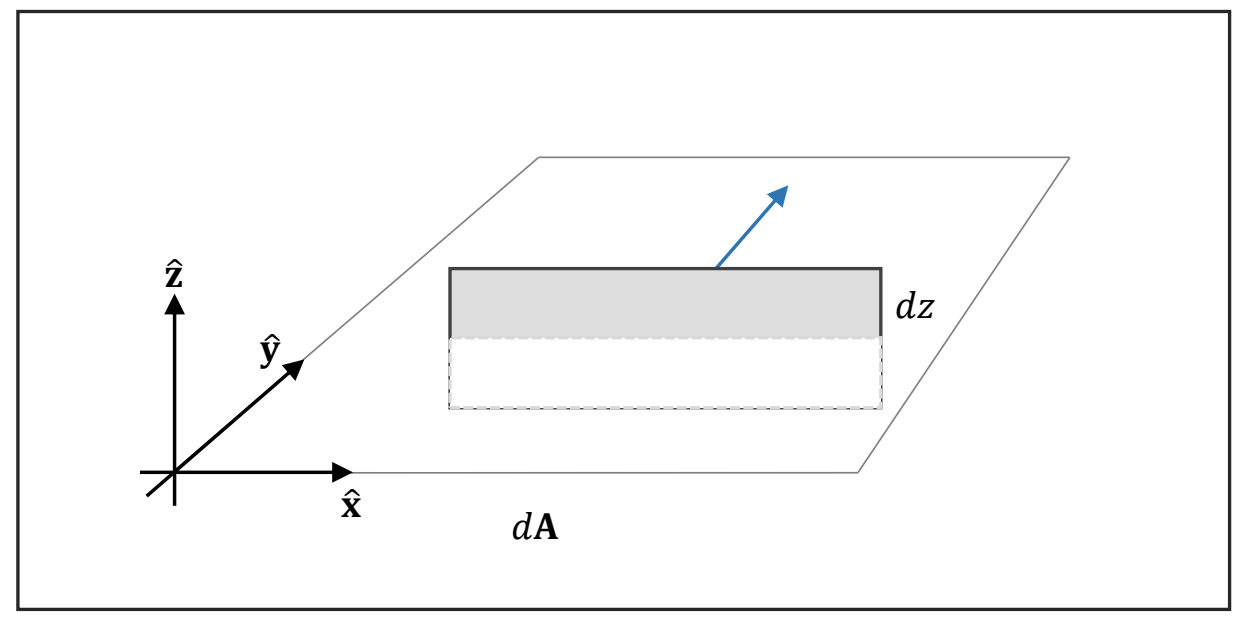

Taking the Proca Equation for the electric field E (3.9), and applying it to this geometry:

$$
\int \boldsymbol{\nabla} \times \mathbf{E} \cdot d \mathbf{A}+\frac{1}{c} \int \frac{\partial \mathbf{B}}{\partial t} \cdot d \mathbf{A}=0
$$

If we then take the limit $d z \rightarrow 0$ (and thus $d A \rightarrow 0$ ), the second term must drop out as $\partial \mathbf{B} / \partial t$ is continuous, since $\mathbf{B}$ cannot change infinitely fast. By Stoke's theorem we are only left with the long ends of the loop,

$$
\oint \mathbf{E} \cdot d \mathbf{l} \rightarrow \int\left(\mathbf{E}^{\prime}-\mathbf{E}\right) \cdot \hat{\mathbf{x}}=0
$$

Equation (3.33) gives us the third boundary condition, 


$$
\mathbf{E}_{\|}^{\prime}-\mathbf{E}_{\|}=0
$$

The fourth Proca equation (3.10), applied to this same geometry, yields the condition for the magnetic field $\mathrm{H}$ with a surface current given by $\mathbf{J}=\mathbf{K} \delta(z)$,

$$
\int \boldsymbol{\nabla} \times \mathbf{H} \cdot d \mathbf{A}-\frac{1}{c} \int \frac{\partial \mathbf{D}}{\partial t} \cdot d \mathbf{A}=\frac{4 \pi}{c} \int \mathbf{K} \cdot d \mathbf{A}-\mu_{\gamma}^{2} \int \mathbf{A} \cdot d \mathbf{A}
$$

In the limit $d z \rightarrow 0$, the time derivative of $\mathbf{D}$ will drop out like before, as will the vector potential term that has been explicitly assumed to be continuous. Applying Stoke's theorem results in,

$$
\oint \mathbf{H} \cdot d \mathbf{l} \rightarrow \int\left(\mathbf{H}^{\prime}-\mathbf{H}\right) \cdot \hat{\mathbf{x}} d x=\frac{4 \pi}{c} \int \mathbf{K} \cdot \hat{\mathbf{x}} d x
$$

The final boundary condition is thus given by,

$$
\mathbf{H}_{\|}^{\prime}-\mathbf{H}_{\|}=\frac{4 \pi}{c} \mathbf{K}_{\|}
$$

With no surface current, the tangential component of $\mathbf{H}$ is therefore also continuous. 


\subsection{ELECTROSTATIC EXPERIMENTS: INVERSE SQUARE LAW}

One of the first serious experimental efforts to test the inverse square law was performed by Cavendish in 1773 [17]. He devised an apparatus made of concentric spheres, an inner conducting sphere, and two outer conducting hemispheres that could be connected and separated via a hinge (Figure 4).

Figure 4: The Cavendish Apparatus (Ref. [7] reproduced with the permission of the IOP)

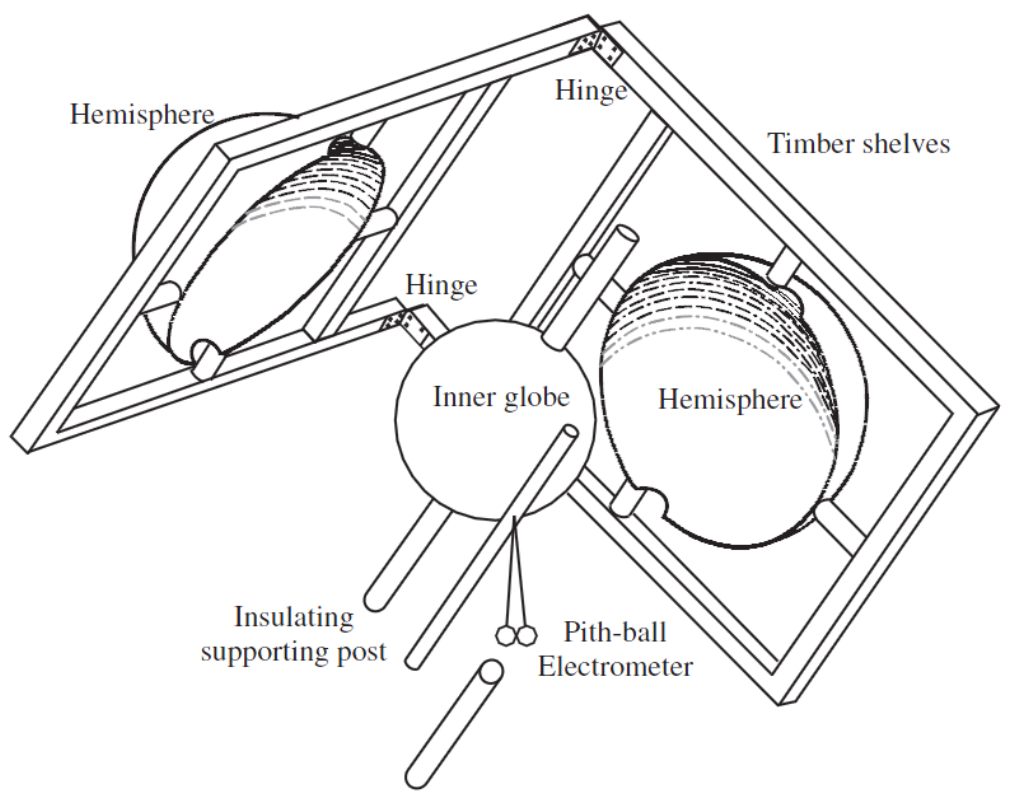

The experiment was performed by closing the outer sphere and connecting it to the inner sphere via a conducting wire. The outer sphere was then charged, after which the connecting wire was severed. The outer sphere was then opened making a measurement of the charge on the inner sphere possible. No charge was found on the inner sphere, which points to an exact inverse square law by the following reasoning: 
Figure 5: Cavendish Experiment Geometry

(Ref. [7] reproduced with the permission of the IOP)

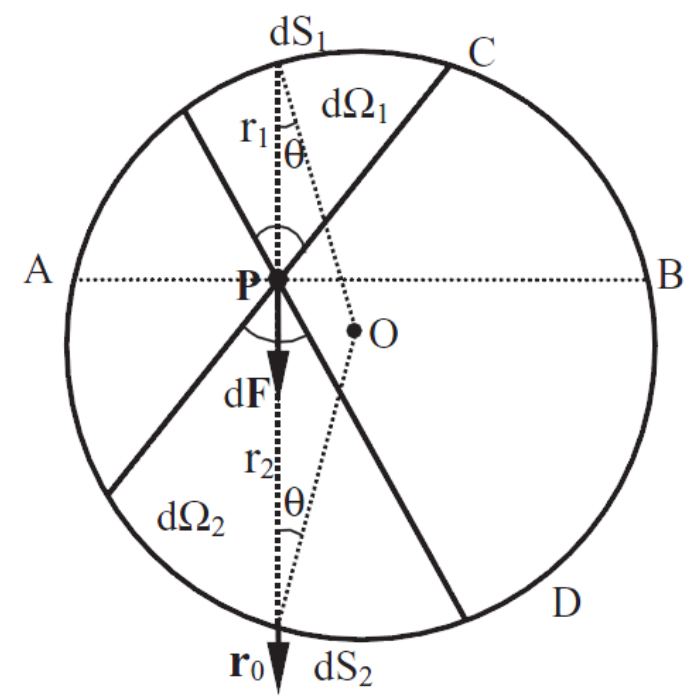

Cavendish argued that if the closed outer sphere possessed a uniform charge density $\sigma$, an arbitrary point $\mathrm{P}$ on the inner sphere would be subject to an electric force $F \propto \sigma S / r^{n}$ where $S$ is the area and $n$ is a number to be determined. By dividing the geometry into two arbitrary parts (Figure 5), and by considering the solid angle of each section, the form for this relation would become,

$$
d \mathbf{F} \propto \frac{\sigma d S_{2}}{r_{2}^{n}} \mathbf{r}_{\mathbf{0}}-\frac{\sigma d S_{1}}{r_{1}^{n}} \mathbf{r}_{\mathbf{0}}=\frac{\sigma d \Omega}{\cos \theta}\left(\frac{1}{r_{2}^{n-2}}-\frac{1}{r_{1}^{n-2}}\right) \mathbf{r}_{\mathbf{0}}
$$

Hence, for an exact value of $n=2$, there is no net force on the point $\mathrm{P}$, thus explaining the null result of the experiment. Cavendish interpreted the results in terms of a deviation from an inverse square law, and concluded for a form $F \propto r^{-(2+\epsilon)}$ that $\epsilon<$ 0.02. As shown in Section 2.3, the correct form for the electric field and thus the electric force when considering a massive photon is given by Equation (2.29). In this 
interpretation, Cavendish's result yields an upper limit for the photon mass of $m_{\gamma}<1 \times$ $10^{-40} \mathrm{~g}$. Maxwell performed this experiment again 100 years later under more careful conditions, and improved the result to find $\epsilon<0.00005$ or $m_{\gamma}<5 \times 10^{-42} \mathrm{~g}$. Many other electrostatic experiments have been performed using different types of apparatus. summarizes some of the results of these experiments.

Figure 6: Coulomb's Law Results (Ref. [7] reproduced with the permission of the IOP)

\begin{tabular}{llll}
\hline Author (year) & Experimental scheme & Deviation $q$ & Limits on $m_{\gamma} \mathrm{g}$ \\
\hline Robison (1769) & $\begin{array}{l}\text { Gravitational torque on a } \\
\text { pivot arm }\end{array}$ & $6 \times 10^{-2}$ & $4 \times 10^{-40}$ \\
Cavendish (1773) & Two concentric metal shells & $2 \times 10^{-2}$ & $1 \times 10^{-40}$ \\
Coulomb (1785) & Torsion balance & $4 \times 10^{-2}$ & $\sim 10^{-39}$ \\
Maxwell (1873) & Two concentric shells & $5 \times 10^{-5}$ & $1 \times 10^{-41}$ \\
Plimpton and Lawton (1936) & Two concentric shells & $2 \times 10^{-9}$ & $3.4 \times 10^{-44}$ \\
Cochran and Franken (1967) & Concentric cubical conductors & $9.2 \times 10^{-12}$ & $3 \times 10^{-45}$ \\
Bartlett et al (1970) & Five concentric shells & $1.3 \times 10^{-13}$ & $3 \times 10^{-46}$ \\
Williams et al (1971) & Five concentric icosahedrons & $(2.7 \pm 3.1) \times 10^{-16}$ & $1.6 \times 10^{-47}$ \\
Fulcher (1985) & Improved result for Williams & $(1.0 \pm 1.2) \times 10^{-16}$ & $1.6 \times 10^{-47}$ \\
Crandall (1983) & experiment & & $8 \times 10^{-48}$ \\
Ryan et al (1985) & Three concentric icosahedrons & $6 \times 10^{-17}$ & $(1.5 \pm 1.4) \times 10^{-42}$ \\
\hline
\end{tabular}

\subsection{MAGNETOSTATIC EXPERIMENTS: VERIFICATION OF AMPERE'S LAW}

A more contemporary measurement testing Ampère's Law was performed by Chernikov et al in 1992 [18]. The integral form of Equation (2.5) in the magnetostatic limit is given by,

$$
\oint \mathbf{B} \cdot d \mathbf{l}=\frac{4 \pi}{C} \int_{\boldsymbol{C}} \mathbf{J} \cdot d \mathbf{S}-\mu_{\gamma}^{2} \int_{\boldsymbol{C}} \mathbf{A} \cdot d \mathbf{S}
$$


where the $\mathbf{J}$ and $\mathbf{A}$ terms are integrated over the area enclosed by the loop $C$. By choosing a geometry such that no current passes through the enclosed area, this simplifies to,

$$
\oint \mathbf{B} \cdot d \mathbf{l}=-\mu_{\gamma}^{2} \int_{\boldsymbol{C}} \mathbf{A} \cdot d \mathbf{S}
$$

Hence, a careful measurement of the magnetic flux will result in an indirect measurement of $\mu_{\gamma}$. Chernikov et al used a sensitive superconducting quantum interference device (SQUID) as a magnetometer to measure the magnetic flux at $1.24 \mathrm{~K}$. Within the uncertainty of the measurement, the null result for the magnetic flux yielded an upper bound of $m_{\gamma}<8.4( \pm 0.8) \times 10^{-46} \mathrm{~g}$.

\subsection{ELECTRODYNAMIC EXPERIMENTS: DISPERSION OF LIGHT}

As shown in Section 2.5, one of the key features of Proca theory is the dispersion of light in a vacuum. In the last few hundred years, the speed of light has been measured by a number of increasingly precise methods. According to special relativity it is a constant in all inertial reference frames, which prompted in 1983 , the exact definition of $c \equiv$ $299,792,458 \mathrm{~m} / \mathrm{s}$, with the meter afterwards becoming a calculated instead of a defined unit [19].

For the purpose of measuring an upper limit to the photon mass, all that needs be shown is a frequency dependence of the speed. Consider two wave packets at different 
frequencies $\omega_{1}$ and $\omega_{2}$, with $\omega_{1}>\omega_{2} \gg \mu_{\gamma} c$ traveling at group velocities $v_{g 1}$ and $v_{g 2}$ respectively. From Equation (2.38), the velocity differential is given by,

$$
\frac{\Delta v}{c}=\frac{v_{g 1}-v_{g 2}}{c} \approx \frac{\mu_{\gamma}^{2} c^{2}}{2}\left(\frac{1}{\omega_{2}^{2}}-\frac{1}{\omega_{1}^{2}}\right) \approx \frac{\mu_{\gamma}^{2}}{8 \pi^{2}}\left(\lambda_{2}^{2}-\lambda_{1}^{2}\right)
$$

Or similarly, if two waves traveling at different frequencies traverse an equivalent distance $L$, the time differential between arrivals is given by,

$$
\Delta t=\frac{L}{v_{g 1}}-\frac{L}{v_{g 2}} \approx \frac{L \mu_{\gamma}^{2}}{8 \pi^{2} c}\left(\lambda_{2}^{2}-\lambda_{1}^{2}\right)
$$

\begin{tabular}{|c|c|c|c|c|}
\hline Author (year) & Type of measurement & $\begin{array}{l}\text { Wavelength (energy or } \\
\text { frequency) range }\end{array}$ & $\frac{\Delta c}{c}$ & Limits on $m_{\gamma} \mathrm{g}$ \\
\hline Ross et al (1937) & $\begin{array}{l}\text { Radio waves transmission } \\
\text { overland }\end{array}$ & $20-120 \mathrm{~m}$ & 0.05 & $5.9 \times 10^{-42}$ \\
\hline $\begin{array}{l}\text { Mandelstam and Papalexi } \\
\text { (1944) }\end{array}$ & $\begin{array}{l}\text { Radio waves transmission } \\
\text { over sea }\end{array}$ & $230-345 \mathrm{~m}$ & $7 \times 10^{-4}$ & $5.0 \times 10^{-43}$ \\
\hline Al'pert et al (1941) & $\begin{array}{l}\text { Radio waves transmission } \\
\text { over sea }\end{array}$ & $300-450 \mathrm{~m}$ & $7 \times 10^{-4}$ & $2.5 \times 10^{-43}$ \\
\hline Florman (1955) & Radio-wave interferometer & $172.8 \mathrm{MHz}-10^{15} \mathrm{~Hz}$ & $10^{-5}$ & $5.7 \times 10^{-42}$ \\
\hline Lovell et al (1964) & $\begin{array}{l}\text { Pulsar observations on } \\
\text { four flare stars }\end{array}$ & $0.54 \mu \mathrm{m}-1.2 \mathrm{~m}$ & $4 \times 10^{-7}$ & $1.6 \times 10^{-42}$ \\
\hline Froome (1958) & Radio-wave interferometer & $72 \mathrm{GHz}$ & $3.3 \times 10^{-7}$ & $4.3 \times 10^{-40}$ \\
\hline Warner et al (1969) & $\begin{array}{l}\text { Observations on Crab } \\
\text { Nebula pulsar }\end{array}$ & $0.35-0.55 \mu \mathrm{m}$ & $5.0 \times 10^{-17}$ & $5.2 \times 10^{-41}$ \\
\hline Brown et al (1973) & Short pulses radiation & $\mathrm{eV}-\mathrm{GeV}$ & $1.8 \times 10^{-6}$ & $1.4 \times 10^{-33}$ \\
\hline Bay et al (1972) & Pulsar emission & Microwave to ultraviolet & $1 \times 10^{-20}$ & $3 \times 10^{-46}$ \\
\hline \multirow[t]{2}{*}{ Schaefer (1999) } & $\begin{array}{l}\text { Gamma ray bursts } \\
\text { (GRB980703) }\end{array}$ & $5.0 \times 10^{9}-1.2 \times 10^{20} \mathrm{~Hz}$ & $6.6 \times 10^{-13}$ & $4.2 \times 10^{-44}$ \\
\hline & $\begin{array}{l}\text { Gamma ray bursts } \\
\text { (GRB930229) }\end{array}$ & $7.2 \times 10^{18}-4.8 \times 10^{19} \mathrm{~Hz}$ & $6.3 \times 10^{-21}$ & $6.1 \times 10^{-39}$ \\
\hline
\end{tabular}

Figure 7: Light Dispersion Results (Ref. [7] reproduced with the permission of the IOP) 
Both (4.4) and (4.5) depend on the square of the wavelength, thus a larger velocity or time differential can be measured by considering low frequencies or long wavelengths. However, low frequency experiments also can introduce considerable imprecision, and so there is a tradeoff. In practice, a wide range of frequencies has been examined, with the results presented in Figure 7. 


\subsection{EXTRA-TERRESTRIAL EXPERIMENTS}

Due to the limits of the previously mentioned terrestrial techniques, most recent improvements to the photon mass upper limit have been achieved by extra-terrestrial experiments. In addition to the dispersion of light, a number of astrophysical phenomena provide the means to calculate an upper limit. The details will not be covered here, with the results presented in Figure 8. A commonly accepted upper limit is on the order of $m_{\gamma}<10^{-49} \mathrm{~g}$

Figure 8: Extra-Terrestrial Experimental Results (Ref. [7] reproduced with the permission of the (OP)

\begin{tabular}{|c|c|c|}
\hline Author (year) & Physical phenomena investigated & Bounds on $m_{\gamma} \mathrm{g}$ \\
\hline De Broglie (1940) & Dispersion of starlight (binary stars) & $8 \times 10^{-40}$ \\
\hline Bass and Schrödinger (1955) & External fields (geomagnetic fields) & $2 \times 10^{-47}$ \\
\hline Yamaguchi (1959) & Scale of hydro-magnetic turbulences in Crab Nebula & $4 \times 10^{-55}$ \\
\hline Gintsburg (1963) & $\begin{array}{l}\text { Altitude-dependence of massive photon } \\
\text { geomagnetic fields }\end{array}$ & $3 \times 10^{-48}$ \\
\hline Patel (1965) & $\begin{array}{l}\text { Dispersion of hydromagnetic waves } \\
\text { (in Earth's magnetosphere) }\end{array}$ & $4 \times 10^{-47}$ \\
\hline \multirow[t]{2}{*}{ Goldhaber and Nieto (1968) } & External fields (geomagnetic fields) & $4 \times 10^{-48}$ \\
\hline & $\begin{array}{l}\text { Altitude-dependence of massive photon } \\
\text { geomagnetic fields }\end{array}$ & $(8-10) \times 10^{-48}$ \\
\hline Feinberg (1969) & Dispersion of starlight (NP0532) & $10^{-44}$ \\
\hline Williams and Park (1971) & Dissipation of large-scale magnetic fields in Galaxy & $3.4 \times 10^{-56}$ \\
\hline Goldhaber and Nieto (1971) & Stability of plasma in Galaxy & $4 \times 10^{-53}$ \\
\hline Byrne and Burman (1972) & Re-examination of Williams and Park's results & $4 \times 10^{-50}$ \\
\hline \multirow[t]{2}{*}{ Byrne and Burman (1973) } & Stability of plasma in Galaxy (for hot intercloud medium) & $10^{-52}$ \\
\hline & Stability of plasma in Galaxy (for cool intercloud medium) & $4 \times 10^{-53}$ \\
\hline Lowenthal (1973) & Gravitational defection for radio source 3C 270 & $7 \times 10^{-40}$ \\
\hline Hollweg (1974) & $\begin{array}{l}\text { Dispersion of hydromagnetic waves } \\
\text { (in interplanetary medium) }\end{array}$ & $1.3 \times 10^{-48}$ \\
\hline Davis et al (1975) & External fields (Jovian magnetic fields) & $8 \times 10^{-49}$ \\
\hline Byrne and Burman (1975) & Mean mass density of the galactic disc & $10^{-51}$ \\
\hline Barnes and Scargle (1975) & Dispersion of hydromagnetic waves (in Crab Nebula) & $3 \times 10^{-54}-3 \times 10^{-53}$ \\
\hline Chibisov (1976) & Analysis of the mechanical stability of the magnetized gas & $3 \times 10^{-60}$ \\
\hline de Bernardis et al (1984) & $\begin{array}{l}\text { Investigation on the spectral behaviour } \\
\text { of the cosmic background dipole anisotropy }\end{array}$ & $(2.9 \pm 0.1) \times 10^{-51}$ \\
\hline Fischbach et al (1994) & External fields (geomagnetic fields) & $1 \times 10^{-48}$ \\
\hline Ryutov (1997) & Analysis of the solar-wind magnetic fields & $10^{-49}$ \\
\hline Goldhaber and Nieto (2003) & Stability of plasma in Coma cluster & $10^{-52}$ \\
\hline Accioly and Paszko (2004) & Gravitational defection of radio waves & $10^{-40}$ \\
\hline
\end{tabular}


The Proca versions of the laws of reflection and refraction (Snell's Law) can be worked out with the foundation material of Chapter 3. The Fresnel equations can in general be worked out as well, though as will be seen, for polarizations other than Type I there are some complications. Neither Type II nor Type III polarized waves will satisfy all boundary conditions, and so a superposition of the two must be considered. The geometry under consideration is shown in Figure 9.

Figure 9: Reflection and Refraction Kinematics

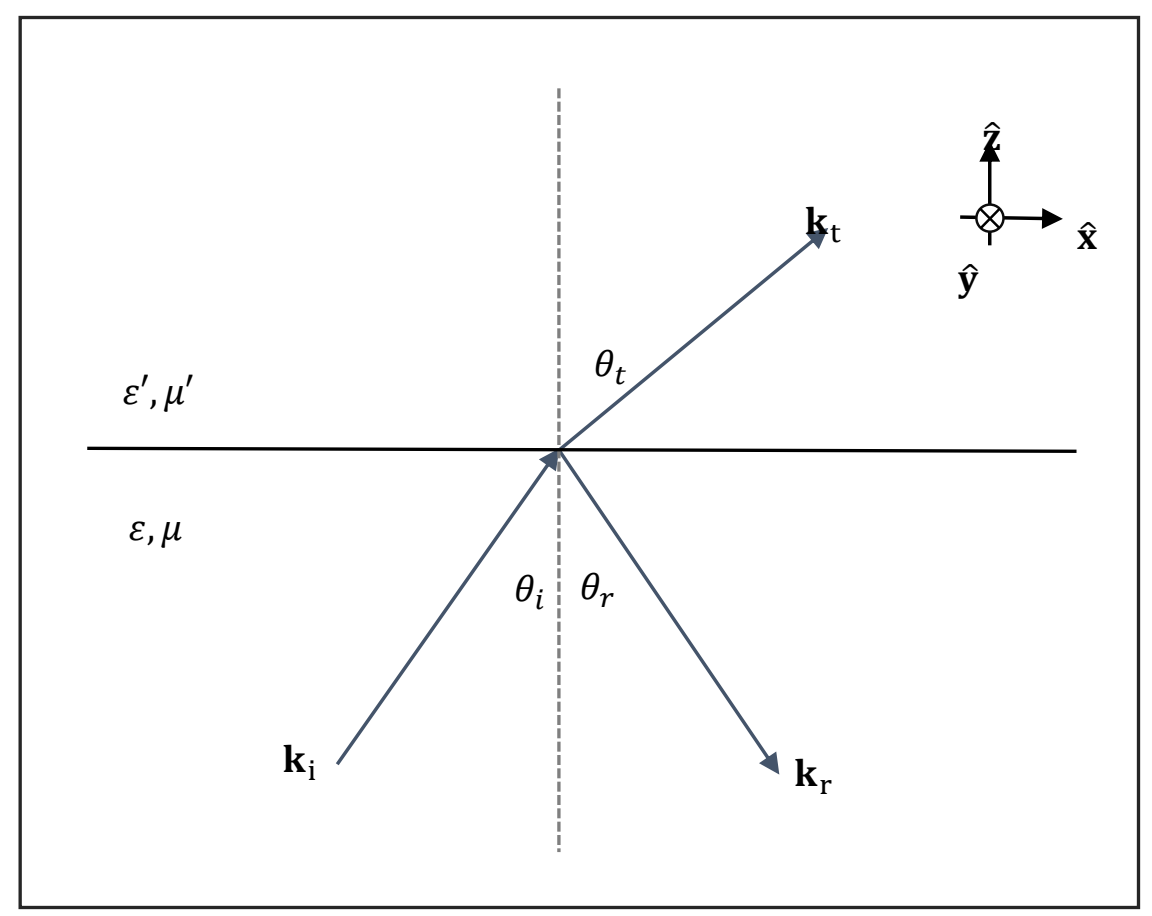

\subsection{KINEMATICS: LAWS OF REFLECTION AND REFRACTION}

The kinematics and dynamics of crossing an interface are worked out, as in the Maxwell case, by matching boundary conditions. The kinematics are derived from the matching of the phase factors, while the dynamics are derived from matching the potentials and 
fields for each polarization (Section 3.2). Beginning with the dynamics, the incident, reflected, and transmitted phase factors can each be matched at the interface,

$$
\left.e^{i\left(\mathbf{k}_{\mathbf{i}} \cdot \mathbf{r}-\omega_{i} t\right)}\right|_{z=0}=\left.e^{i\left(\mathbf{k}_{\mathbf{r}} \cdot \mathbf{r}-\omega_{r} t\right)}\right|_{z=0}=\left.e^{i\left(\mathbf{k}_{\mathbf{t}} \cdot \mathbf{r}-\omega_{t} t\right)}\right|_{z=0}
$$

For these to hold for an arbitrary time $t$, the frequencies must all be equivalent,

$$
\omega_{i}=\omega_{r}=\omega_{t}=\omega
$$

Thus all of the spatial components must match,

$$
\left.\mathbf{k}_{\mathbf{i}} \cdot \mathbf{r}\right|_{z=0}=\left.\mathbf{k}_{\mathbf{r}} \cdot \mathbf{r}\right|_{z=0}=\left.\mathbf{k}_{\mathbf{t}} \cdot \mathbf{r}\right|_{z=0}
$$

The geometry has been chosen such that $k_{i y}=k_{r y}=k_{t y}=0$, therefore,

$$
k_{i} \sin \theta_{i}=k_{r} \sin \theta_{r}=k_{t} \sin \theta_{t}
$$

The wave numbers in the different regions will in general follow different dispersion relations due to the different values for $\varepsilon$ and $\mu$. However the incident and the reflected wave will necessarily follow the same dispersion relation and so have the same wave number magnitude. For simplicity we will call $k=k_{i}=k_{r}$. From Equation (5.4), this implies the law of reflection still holds in Proca theory, 


$$
\theta_{i}=\theta_{r}=\theta
$$

The law of refraction (Snell's Law) is given by Equation (5.4), combined with this result. Using simpler notation, $k_{t}=k^{\prime}$ and $\theta_{t}=\theta^{\prime}$,

$$
k \sin \theta=k^{\prime} \sin \theta^{\prime}
$$

Unlike the Maxwell case, this cannot be simplified further by introducing the constant indices of refraction $n \equiv \sqrt{\varepsilon \mu}$ and $n^{\prime} \equiv \sqrt{\varepsilon^{\prime} \mu^{\prime}}$, as these are only possible by using the simpler Maxwell dispersion relation of $k^{2}=\varepsilon \mu \omega^{2} / c^{2}$. The Proca dispersion relations do not allow this simplification. To see the deviation of Equation (5.6) from Snell's Law more straightforwardly, the dispersion relations can be re-written as,

$$
k= \begin{cases}\frac{n \omega}{c} \sqrt{1-\frac{\mu_{\gamma}^{2} c^{2}}{\varepsilon \omega^{2}}} \approx \frac{n \omega}{c}\left(1-\frac{\mu_{\gamma}^{2} c^{2}}{2 \varepsilon \omega^{2}}\right) & (\text { Type I,II) } \\ \frac{\omega}{c} \sqrt{1-\frac{\mu_{\gamma}^{2} c^{2}}{\varepsilon \omega^{2}}} \approx \frac{\omega}{c}\left(1-\frac{\mu_{\gamma}^{2} c^{2}}{2 \varepsilon \omega^{2}}\right) & \text { (Type III) }\end{cases}
$$

Equation (5.6) can be re-written with these forms for the transverse modes,

$$
\frac{n \sin \theta}{n^{\prime} \sin \theta^{\prime}} \approx\left(1-\frac{\mu_{\gamma}^{2} c^{2}}{2 \varepsilon \omega^{2}}\right) /\left(1-\frac{\mu_{\gamma}^{2} c^{2}}{2 \varepsilon^{\prime} \omega^{2}}\right) \approx 1+\frac{\mu_{\gamma}^{2} c^{2}}{2 \omega^{2}}\left(\frac{1}{\varepsilon^{\prime}}-\frac{1}{\varepsilon}\right)
$$


In the massless limit, Type I and II smoothly resolve to Snell's Law. As in the case of dispersion measurements, the most useful form of an experiment would be for low frequency light, which would exacerbate the photon mass effect as can be seen from Equation (5.8).

From Equation (5.7) it can be also seen that Type III will not resemble Snell's Law, even in the massless limit. In this case there will be no refraction, with $\theta=\theta^{\prime}$. However as has already been shown, Type III longitudinal waves do not exist in Maxwell theory, so this should not cause alarm. 


\subsection{DYNAMICS: TYPE I FRESNEL EQUATIONS}

To work out the full dynamics of the effects of reflection and refraction, the boundary conditions of Section 3.3 must be applied to the Type I potentials and fields of Section 3.2.

Figure 10: Type I Dynamics

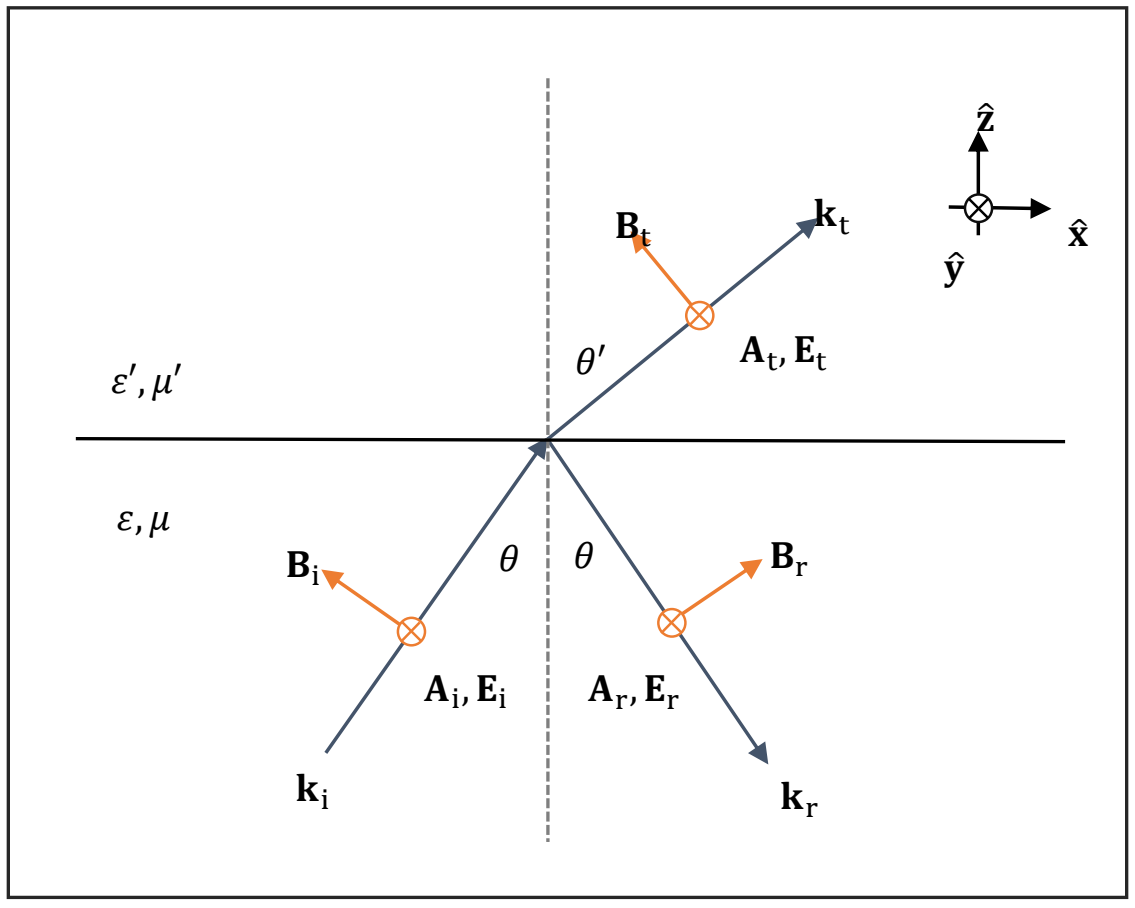

For this polarization, the only non-zero potential component is $A_{y}$.

$$
A_{i}+A_{r}=A_{t}
$$

The boundary conditions for $E_{\|}$and $B_{\perp}$, with the use of Snell's Law (5.6) replicate this result. Since in this polarization, $D_{\perp}=0$, the only other independent boundary condition is given by the continuity of $H_{\|}$, 


$$
H_{i x}+H_{r x}=H_{t x} \quad \rightarrow \quad-\frac{i k A_{i}}{\mu} \cos \theta+\frac{i k A_{r}}{\mu} \cos \theta=-\frac{i k^{\prime} A_{t}}{\mu^{\prime}} \cos \theta^{\prime}
$$

Using our result from the potential boundary condition (5.9), this can be re-arranged as,

$$
\begin{aligned}
\frac{k}{\mu}\left(A_{i}-A_{r}\right) \cos \theta & =\frac{k^{\prime}}{\mu^{\prime}}\left(A_{i}+A_{r}\right) \cos \theta^{\prime} \\
\Rightarrow \quad\left(\frac{k}{\mu} \cos \theta-\frac{k^{\prime}}{\mu^{\prime}} \cos \theta^{\prime}\right) A_{i} & =\left(\frac{k}{\mu} \cos \theta+\frac{k^{\prime}}{\mu^{\prime}} \cos \theta^{\prime}\right) A_{r}
\end{aligned}
$$

And by applying Snell's Law (5.6) to this result,

$$
\begin{gathered}
\cos \theta^{\prime}=\sqrt{1-\sin ^{2} \theta^{\prime}}=\sqrt{1-\frac{k^{2}}{k^{\prime 2}} \sin ^{2} \theta}=\frac{1}{k^{\prime}} \sqrt{k^{\prime 2}-k^{2} \sin ^{2} \theta} \\
\Rightarrow \quad \frac{A_{r}}{A_{i}}=\frac{k \cos \theta-\frac{\mu}{\mu^{\prime}} \sqrt{k^{\prime 2}-k^{2} \sin ^{2} \theta}}{k \cos \theta+\frac{\mu}{\mu^{\prime}} \sqrt{k^{\prime 2}-k^{2} \sin ^{2} \theta}}
\end{gathered}
$$

Equation (5.11) is the Proca-Fresnel Equation for Type I reflection. The corresponding ratio for the strength of refracted waves can be obtained by solving for the other variable $A_{t}$ in Equation (5.10),

$$
\begin{aligned}
& \frac{k}{\mu}\left(A_{i}-A_{t}+A_{i}\right) \cos \theta=\frac{k^{\prime}}{\mu^{\prime}}\left(A_{t}\right) \cos \theta^{\prime} \\
\Rightarrow \quad & 2 \frac{k}{\mu} \cos \theta A_{i}=\left(\frac{k}{\mu} \cos \theta+\frac{k^{\prime}}{\mu^{\prime}} \cos \theta^{\prime}\right) A_{t}
\end{aligned}
$$


And applying Snell's Law once again, we arrive at the Proca-Fresnel Equation for Type I refraction,

$$
\Rightarrow \quad \frac{A_{t}}{A_{i}}=\frac{2 k \cos \theta}{k \cos \theta+\frac{\mu}{\mu^{\prime}} \sqrt{k^{\prime 2}-k^{2} \sin ^{2} \theta}}
$$

Both equations (5.11) and (5.12) resolve to the Maxwell-Fresnel equations for s-polarized waves by taking the massless limit $k \rightarrow n \omega / c$,

$$
\begin{aligned}
& \frac{A_{r}}{A_{i}}=\frac{E_{r}}{E_{i}} \rightarrow \frac{n \cos \theta-\frac{\mu}{\mu^{\prime}} \sqrt{n^{\prime 2}-n^{2} \sin ^{2} \theta}}{n \cos \theta+\frac{\mu}{\mu^{\prime}} \sqrt{n^{\prime 2}-n^{2} \sin ^{2} \theta}} \\
& \frac{A_{t}}{A_{i}}=\frac{E_{t}}{E_{i}} \rightarrow \frac{2 n \cos \theta}{n \cos \theta+\frac{\mu}{\mu^{\prime}} \sqrt{n^{\prime 2}-n^{2} \sin ^{2} \theta}}
\end{aligned}
$$

Just as with the Proca-Snell law, the form of the Proca-Fresnel equations is identical to the Maxwell-Fresnel case, and any deviation due to the massive photon is contained within the dispersion relation. As will be seen, this is only true for the Type I polarization. Type III has no counterpart in Maxwell theory, but Type II is equitable with the $\mathrm{p}$-wave, and so it is surprising that even this form will differ in Proca theory. 


\subsection{DYNAMICS: TYPE // FRESNEL EQUATIONS}

The Type II polarization can be approached in the same way, first matching the potential boundary condition. In this case there will be both a non-zero $A_{x}$ and $A_{z}$ component, with $A_{y}$ and $\Phi$ both equal to zero in this polarization,

Figure 11: Type II Dynamics

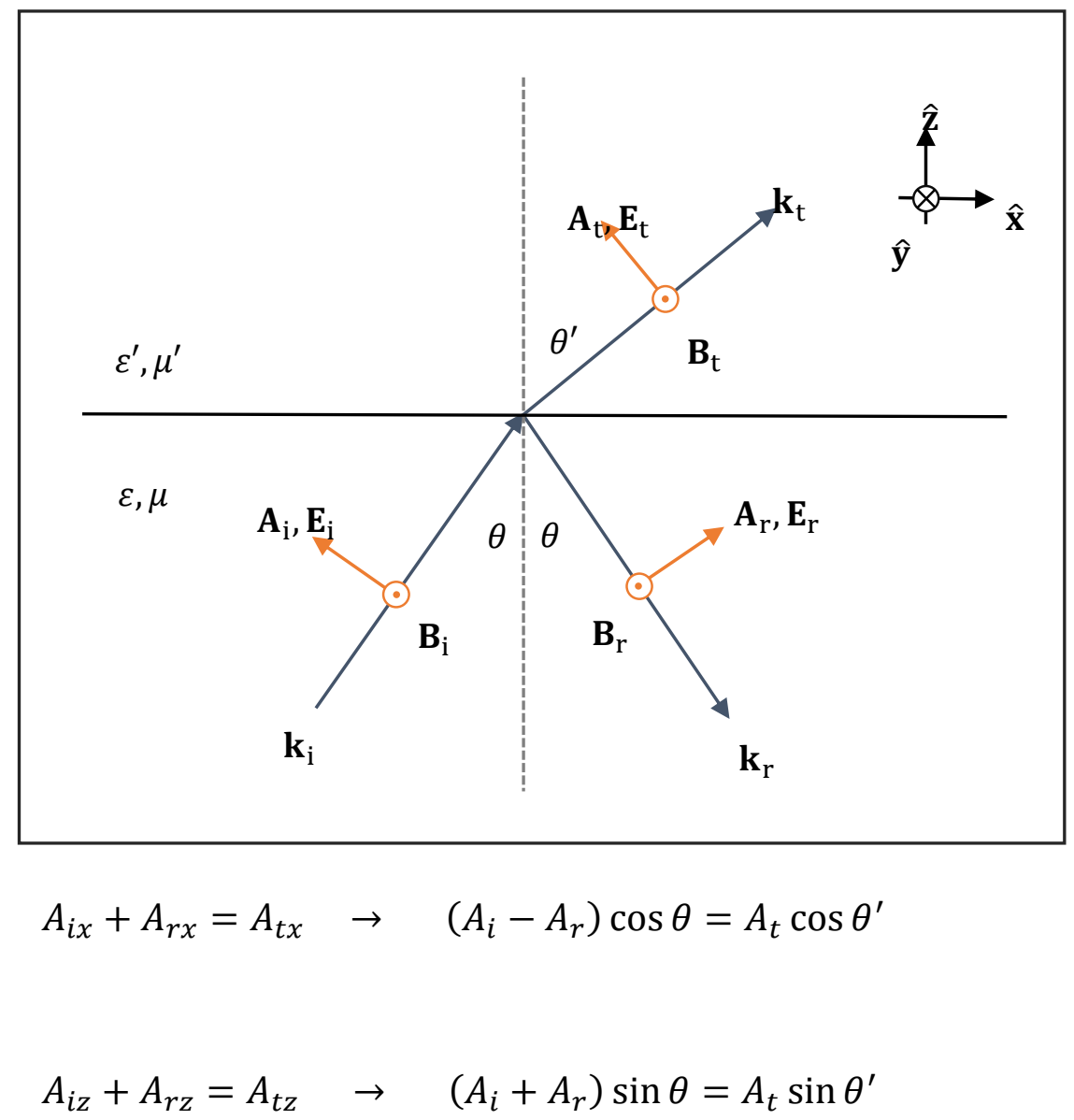

Equations (5.15) and (5.16) together are sufficient to determine the Type II Proca-Fresnel equations. However, by first checking the field conditions we can see that this 
polarization cannot satisfy all boundary conditions. The $\mathbf{E}_{\|}$condition is redundant and simply re-produces (5.15). $\mathbf{B}_{\perp}$ is zero for all waves. $\mathbf{D}_{\perp}$ and $\mathbf{H}_{\|}$conditions result in,

$$
\begin{aligned}
& D_{i z}+D_{r z}=D_{t z} \quad \rightarrow \quad \varepsilon\left(A_{i}+A_{r}\right) \sin \theta=\varepsilon^{\prime} A_{t} \sin \theta^{\prime} \\
& H_{i y}+H_{r y}=H_{t y} \quad \rightarrow \quad \frac{k}{\mu}\left(A_{i}+A_{r}\right)=\frac{k^{\prime}}{\mu^{\prime}} A_{t}
\end{aligned}
$$

In the Maxwell case, equations (5.17) and (5.18) are equivalent through the dispersion relation. The only other condition applied is that for $\mathbf{E}_{\|}$which is equivalent to (5.15). This is enough to derive the Maxwell-Fresnel equations. However in the Proca case, equations (5.17) and (5.18) are not equivalent, and both at odds with equations (5.15) and (5.16). Hence, the Type II polarization does not satisfy all boundary condition. A similar situation can be shown for the Type III longitudinal polarization.

What is required is to construct linear combinations of Type II and Type III which do satisfy all boundary conditions. This has been done in both [10] and [11]. In both these investigations, the Casimir force with a massive photon was the phenomenon under consideration. Therein, the Type I polarization was found to satisfy all boundary conditions as in the Maxwell case and in the above work, and was deemed the TE mode. The Type II and Type III polarizations however needed to be considered together, and two linear combinations of them were found to satisfy all necessary conditions. Together these were termed the TM mode(s). In the massless limit, the Type III polarization 
vanishes, and the linear combinations of these polarizations converged to the ordinary Maxwell TM mode as expected. The mathematical work done there-in will not be reproduced here, but the results of these studies into the Proca-Casimir force would be straightforwardly extensible to optics. 
Most of the current studies for probing possible photon mass effects to date have been limited to optical fields of macroscopic systems. These include both far fields and near fields ranging from the electrostatic and dynamic experiments described in Chapter 4, to the Casimir force fields investigated by [10] and [11], as well as the results from the preceding chapter. In this chapter we would like to explore a different approach from studying such effects on atomic spectroscopy, since high precession measurements in spectroscopy have been well established. As a first attempt, we shall focus as an example on the $21 \mathrm{~cm}$ radiation, one of the most important spectral lines in astronomy given the abundance of Hydrogen in both galactic and inter-galactic regions. This spectral line is caused by the energy difference between parallel and anti-parallel spin states for the proton and electron.

\subsection{CM RADIATION IN THE MASSLESS LIMIT}

The spin-spin interaction will depend on the magnetic moments of the proton and electron,

$$
\begin{aligned}
& \boldsymbol{\mu}_{e}=-\frac{g_{e} e}{2 c m_{e}} \mathbf{S}_{e} \\
& \boldsymbol{\mu}_{p}=+\frac{g_{p} e}{2 c m_{p}} \mathbf{S}_{p}
\end{aligned}
$$


These magnetic moments will depend on the masses of the particles $m$, the spins $\mathbf{S}$, and the g-factors, $g_{e}=2.00231930436153$ and $g_{p}=5.585694713$ [20]. The magnetic field experienced by the proton due to the magnetic moment of the electron will be given by the standard dipole field,

$$
\mathbf{B}_{e}^{(2)}=\frac{8 \pi}{3} \boldsymbol{\mu}_{e} \delta(\mathbf{r})+\frac{3\left(\boldsymbol{\mu}_{e} \cdot \mathbf{r}\right) \mathbf{r}-r^{2} \boldsymbol{\mu}_{e}}{r^{5}}
$$

The hyperfine Hamiltonian will be given by the interaction between the proton magnetic moment and the magnetic field due to the electron moment,

$$
\widehat{H}_{h f}=-\boldsymbol{\mu}_{p} \cdot \mathbf{B}_{e}^{(2)}
$$

The corresponding energy for the ground state of Hydrogen will then be given by,

$$
E_{h f}=\left\langle\psi_{100}\left|\widehat{H}_{h f}\right| \psi_{100}\right\rangle
$$

The ground state of the Hydrogen is given by the familiar wave function,

$$
\psi_{100}(r)=\frac{1}{\sqrt{\pi a_{0}^{3}}} e^{-r / a_{0}}
$$

The Bohr radius $a_{0}=\hbar^{2} / m_{e} e^{2}$ is used here. Since the ground state is spherically symmetric, as will be shown in Section 6.3, the integration over the second non-singular term of equation (6.3) will be zero, leaving us with only, 


$$
E_{h f}=-\frac{8 \pi}{3}\left(\boldsymbol{\mu}_{p} \cdot \boldsymbol{\mu}_{e}\right)\left|\psi_{100}(0)\right|^{2}=\frac{2 g_{e} g_{p} e^{2}}{3 m_{e} m_{p} c^{2} a_{0}^{3}}\left(\mathbf{S}_{p} \cdot \mathbf{S}_{e}\right)
$$

The spin-spin interaction term for spin $1 / 2$ particles can be expressed using the quantum numbers $f$,

$$
\mathbf{S}_{p} \cdot \mathbf{S}_{e}=\frac{\hbar^{2}}{2}\left(f(f+1)-\frac{3}{2}\right)
$$

For parallel spins, $f=1$, and for antiparallel spins $f=0$. Thus, for the energy transition between parallel and anti-parallel spin configurations,

$$
\Delta E_{h f}=\frac{2 g_{e} g_{p} e^{2} \hbar^{2}}{3 m_{e} m_{p} c^{2} a_{0}^{3}}=\frac{2}{3}\left(\frac{m_{e}}{m_{p}}\right) g_{e} g_{p} m_{e} c^{2} \alpha^{4}
$$

The fine structure constant is given by $\alpha=e^{2} / \hbar c$. The energy difference (6.9), as well as accounting for higher order terms, yields the famous result:

$$
\begin{gathered}
f_{h f}=\Delta E_{h f} / h=1420.40575177 \mathrm{MHz} \\
\lambda_{h f}=c / f_{h f}=21.10611405413 \mathrm{~cm}
\end{gathered}
$$

A few of these steps will need to be carefully re-evaluated to incorporate a non-zero photon mass. The dipole expression (6.3) will change as well as the ground state wave function (6.6). 


\subsection{GROUND STATE WAVE FUNCTION FOR THE YUKAWA POTENTIAL}

The ground state wavefunction (6.6) was calculated for an electron bound to a proton subject to a Coulomb potential of the form $V(r)=-e^{2} / r$. By considering a massive photon, as shown in Section 2.3, a Yukawa potential should instead be considered:

$$
V(r)=-\frac{e^{2}}{r} e^{-\mu_{\gamma} r}
$$

Exact analytic solutions for the Yukawa wavefunctions are extremely complicated, but the work of Garavelli et al [13] has come close using an iterative approach that closely agrees with variational and numerical methods. The approximate analytic form they found for the ground state is:

$$
\psi_{100}(\rho)=A e^{-\alpha \rho}\left(\frac{1-e^{-\beta \rho}}{\beta \rho}\right)
$$

Where $A$ is a normalization constant and the dimensionless variable $\rho$ and parameters $\alpha$, $\beta, \gamma$, and $\delta$ are given by the following relations:

$$
\begin{aligned}
& \rho=r / a_{0} \\
& \delta=a_{0} \mu_{\gamma} \\
& \alpha=-\gamma\left[1+\frac{4(\alpha+\gamma)}{(\alpha-\gamma)^{2}} \ln \left(\frac{(2 \alpha+\delta)(2 \gamma+\delta)}{(\alpha+\gamma+\delta)^{2}}\right)\right]
\end{aligned}
$$




$$
\begin{aligned}
& \beta=\gamma-\alpha \\
& \gamma=\frac{1}{2}[\alpha+\delta+\sqrt{(\alpha+\delta)(\alpha+5 \delta)}]
\end{aligned}
$$

These expressions can be simplified by taking the lowest order correction in $\delta$.

$$
\begin{aligned}
\gamma & \approx \frac{1}{2}\left[\alpha+\delta+\alpha \sqrt{1+\frac{6 \delta}{\alpha}}\right] \approx \frac{1}{2}\left[\alpha+\delta+\alpha\left(1+\frac{3 \delta}{\alpha}\right)\right]=\alpha+2 \delta \\
& \Rightarrow \beta \approx 2 \delta \\
\alpha & \approx-\gamma\left[1+\frac{4(\alpha+\gamma)}{(\alpha-\gamma)^{2}}\left(\left(\frac{4 \alpha \gamma+2(\alpha+\gamma) \delta+\delta^{2}}{(\alpha+\gamma)^{2}+2(\alpha+\gamma) \delta+\delta^{2}}\right)-1\right)\right] \\
& =-\gamma\left[1+\frac{4(\alpha+\gamma)}{(\alpha-\gamma)^{2}}\left(\frac{-(\alpha-\gamma)^{2}}{(\alpha+\gamma)^{2}+2(\alpha+\gamma) \delta+\delta^{2}}\right)\right] \\
& \approx-\gamma\left[1+\frac{4(\alpha+\gamma)}{(\alpha-\gamma)^{2}} \cdot \frac{-(\alpha-\gamma)^{2}}{(\alpha+\gamma)(\alpha+\gamma+2 \delta)}\right]=-\gamma\left[1-\frac{4}{(\alpha+\gamma+2 \delta)}\right]
\end{aligned}
$$

Further simplification is possible by substituting the approximate form for $\gamma$ (6.19),

$$
\alpha \approx-(\alpha+2 \delta)\left(1-\frac{2}{(\alpha+2 \delta)}\right)=-\alpha-2 \delta+2 \rightarrow \alpha \approx 1-\delta
$$

Substituting (6.20) and (6.21), the ground state wave function to first order in $\delta$ in dimensionless units is given by, 


$$
\psi_{100}(\rho)=A e^{-(1-\delta) \rho}\left(\frac{1-e^{-2 \delta \rho}}{2 \delta \rho}\right)
$$

In standard units, this ground state function is,

$$
\psi_{100}(r)=A e^{-\frac{1}{a_{0}}(1-\delta) r}\left(\frac{1-e^{-2 \mu_{\gamma} r}}{2 \mu_{\gamma} r}\right)
$$

It will next be necessary to normalize this approximate ground state function, performed in the usual way by integrating over all space.

$$
\begin{aligned}
1 & =\int\left|\psi_{100}(r)\right|^{2} r^{2} d r d \Omega=\frac{\pi A^{2}}{\mu_{\gamma}^{2}} \int_{0}^{\infty} e^{-2\left(1-a_{0} \mu_{\gamma}\right) r / a_{0}}\left(1-e^{-2 \mu_{\gamma} r}\right)^{2} d r \\
& =\frac{\pi A^{2}}{\mu_{\gamma}^{2}} \int_{0}^{\infty} e^{-\frac{2 r}{a_{0}}(1-\delta)}-2 e^{-\frac{2 r}{a_{0}}}+e^{-\frac{2 r}{a_{0}}(1+\delta)} d r= \\
& =-\frac{\pi a_{0} A^{2}}{2 \mu_{\gamma}^{2}}\left[\frac{1}{1-\delta} e^{-\frac{2 r}{a_{0}}(1-\delta)}-2 e^{-\frac{2 r}{a_{0}}}+\frac{1}{1+\delta} e^{-\frac{2 r}{a_{0}}(1+\delta)}\right]_{0}^{\infty} \\
& =\frac{\pi a_{0} A^{2}}{2 \mu_{\gamma}^{2}}\left[\frac{1}{1-\delta}-2+\frac{1}{1+\delta}\right]=\frac{\pi a_{0} A^{2}}{2 \mu_{\gamma}^{2}}\left(\frac{2 \delta^{2}}{1-\delta^{2}}\right)=\frac{\pi a_{0}{ }^{3} A^{2}}{1-a_{0}{ }^{2} \mu_{\gamma}^{2}} \\
& \rightarrow A^{2}=\frac{1}{\pi a_{0}{ }^{3}}\left(1-a_{0}{ }^{2} \mu_{\gamma}^{2}\right)
\end{aligned}
$$

\subsection{MAGNETIC DIPOLE FIELD FOR A MASSIVE PHOTON}

Dragulin and Leung [12] showed through the method of a Green dyadic for the Proca fields that the correct form for the magnetic dipole field is given by, 


$$
\mathbf{B}_{e}^{(2)}=\frac{8 \pi}{3} \boldsymbol{\mu}_{e} \delta(\mathbf{r})+\frac{e^{-\mu_{\gamma} r}}{r^{5}}\left[\left(3+3 \mu_{\gamma} r+\mu_{\gamma}^{2} r^{2}\right)\left(\boldsymbol{\mu}_{e} \cdot \mathbf{r}\right) \mathbf{r}-r^{2}\left(1+\mu_{\gamma} r+\mu_{\gamma}^{2} r^{2}\right) \boldsymbol{\mu}_{e}\right]
$$

In the massless limit, this can be seen to converge to (6.3). In the massless limit the nonsingular term vanishes due to spherical symmetry. We will have to carefully check the integral of the non-singular term of $(6.25)$ to see if this is still the case. The argument stems from the identity,

$$
\int \frac{x_{i} x_{j}}{r^{2}} d \Omega=\frac{4 \pi}{3} \delta_{i j}
$$

This identity can be verified through direct integration or by invoking spherical harmonics [21], [9]. To see how this is of use we will work out the details of the integral (6.5) for the non-singular terms of the Proca field (6.25). A spherically-symmetric ground state wavefunction $\psi_{100}$, will be assumed, the exact form of which is given by (6.23).

$$
\begin{aligned}
& \left\langle\psi_{100}\left|\frac{e^{-\mu_{\gamma} r}}{r^{5}}\left(3+3 \mu_{\gamma} r+\mu_{\gamma}^{2} r^{2}\right) \mu_{e i} x_{i} \mu_{p j} x_{j}-\frac{e^{-\mu_{\gamma} r}}{r^{3}}\left(1+\mu_{\gamma} r+\mu_{\gamma}^{2} r^{2}\right) \mu_{e i} \mu_{p i}\right| \psi_{100}\right\rangle \\
= & \int\left|\psi_{100}\right|^{2} \frac{e^{-\mu_{\gamma} r}}{r}\left[\left(3+3 \mu_{\gamma} r+\mu_{\gamma}^{2} r^{2}\right) \mu_{e i} \mu_{p j} \int \frac{x_{i} x_{j}}{r^{2}} d \Omega-4 \pi\left(1+\mu_{\gamma} r+\mu_{\gamma}^{2} r^{2}\right) \mu_{e i} \mu_{p i}\right] d r \\
= & \int\left|\psi_{100}\right|^{2} \frac{e^{-\mu_{\gamma} r}}{r}\left[4 \pi\left(1+\mu_{\gamma} r+\frac{1}{3} \mu_{\gamma}^{2} r^{2}\right) \mu_{e i} \mu_{p j} \delta_{i j}-4 \pi\left(1+\mu_{\gamma} r+\mu_{\gamma}^{2} r^{2}\right) \mu_{e i} \mu_{p i}\right] d r \\
= & 4 \pi\left(\boldsymbol{\mu}_{p} \cdot \boldsymbol{\mu}_{e}\right) \int\left|\psi_{100}\right|^{2} \frac{e^{-\mu_{\gamma} r}}{r}\left(-\frac{2}{3} \mu_{\gamma}^{2} r^{2}\right) d r=-\frac{8 \pi}{3} \mu_{\gamma}^{2}\left(\boldsymbol{\mu}_{p} \cdot \boldsymbol{\mu}_{e}\right) \int\left|\psi_{100}\right|^{2} r e^{-\mu_{\gamma} r} d r
\end{aligned}
$$

This integral can be performed by substituting the wavefunction (6.23), 


$$
\begin{aligned}
& \int\left|\psi_{100}\right|^{2} r e^{-\mu_{\gamma} r} d r=\frac{A^{2}}{4 \mu_{\gamma}^{2}} \int_{0}^{\infty} \frac{1}{r} e^{-\frac{2}{a_{0}}(1-\delta) r}\left(1-e^{-2 \mu_{\gamma} r}\right)^{2} e^{-\mu_{\gamma} r} d r \\
& =\frac{A^{2}}{4 \mu_{\gamma}^{2}} \int_{0}^{\infty} \frac{1}{r} e^{-\frac{1}{a_{0}}(2-\delta) r}\left(1-2 e^{-\frac{2}{a_{0}} \delta r}+e^{-\frac{4}{a_{0}} \delta r}\right) d r \\
& =\frac{A^{2}}{4 \mu_{\gamma}^{2}} \int_{0}^{\infty} \frac{1}{r}\left(e^{-\frac{1}{a_{0}}(2-\delta) r}-2 e^{-\frac{1}{a_{0}}(2+\delta) r}+e^{-\frac{1}{a_{0}}(2+3 \delta) r}\right) d r \\
& =\frac{A^{2}}{4 \mu_{\gamma}^{2}}\left[\int_{0}^{\infty} \frac{1}{r}\left(e^{-\frac{1}{a_{0}}(2-\delta) r}-e^{-\frac{1}{a_{0}}(2+\delta) r}\right) d r+\int_{0}^{\infty} \frac{1}{r}\left(e^{-\frac{1}{a_{0}}(2+3 \delta) r}-e^{-\frac{1}{a_{0}}(2+\delta) r}\right) d r\right]
\end{aligned}
$$

This can be solved exactly by the special integral $\int_{0}^{\infty} \frac{1}{u}\left(e^{-a u}-e^{-b u}\right) d u=\ln (b / a)$,

$$
\begin{aligned}
& =\frac{A^{2}}{4 \mu_{\gamma}^{2}}\left[\ln \left(\frac{2+\delta}{a_{0}} \cdot \frac{a_{0}}{2-\delta}\right)+\ln \left(\frac{2+\delta}{a_{0}} \cdot \frac{a_{0}}{2+3 \delta}\right)\right] \approx \frac{A^{2}}{4 \mu_{\gamma}^{2}}\left(\frac{2+\delta}{2-\delta}-1+\frac{2+\delta}{2+3 \delta}-1\right) \\
& =\frac{A^{2}}{4 \mu_{\gamma}^{2}}\left(\frac{8 \delta^{2}}{4+4 \delta-3 \delta^{2}}\right)=\frac{1}{2} a_{0}{ }^{2} A^{2}\left(1+\delta-\frac{3}{4} \delta^{2}\right)^{-1} \approx \frac{1}{2} a_{0}{ }^{2} A^{2}
\end{aligned}
$$

As will be seen in the subsequent section, the higher order terms of $\delta$ in (6.27) can be dropped to be consistent with the order of precision of other approximations. Thus, the integration of the non-singular term is non-zero, with the remainder given by,

$$
\approx-\frac{4 \pi}{3}\left(\boldsymbol{\mu}_{p} \cdot \boldsymbol{\mu}_{e}\right) a_{0}^{2} \mu_{\gamma}^{2} A^{2}
$$




\subsection{REVISION TO THE 21 CM RADIATION IN PROCA THEORY}

The necessary revision to the massless case then will come in the form of the groundstate normalization constant (6.24) and the non-singular term (6.28). Combining these terms we find,

$$
\begin{aligned}
& E_{h f}=-\frac{8 \pi}{3}\left(\boldsymbol{\mu}_{p} \cdot \boldsymbol{\mu}_{e}\right)\left[\left|\psi_{100}(0)\right|^{2}-\mu_{\gamma}^{2} \int\left|\psi_{100}\right|^{2} r e^{-\mu_{\gamma} r} d r\right] \\
& \cong-\frac{8}{3 a_{0}^{3}}\left(\boldsymbol{\mu}_{p} \cdot \boldsymbol{\mu}_{e}\right)\left(1-a_{0}^{2} \mu_{\gamma}^{2}\right)\left[1-\frac{1}{2} a_{0}^{2} \mu_{\gamma}^{2}\left(1+a_{0} \mu_{\gamma}-\frac{3}{4} a_{0}^{2} \mu_{\gamma}^{2}\right)^{-1}\right] \\
& \approx-\frac{8}{3 a_{0}^{3}}\left(\boldsymbol{\mu}_{p} \cdot \boldsymbol{\mu}_{e}\right)\left(1-a_{0}^{2} \mu_{\gamma}^{2}\right)\left(1-\frac{1}{2} a_{0}^{2} \mu_{\gamma}^{2}\right) \approx-\frac{8}{3 a_{0}{ }^{3}}\left(\boldsymbol{\mu}_{p} \cdot \boldsymbol{\mu}_{e}\right)\left(1-\frac{3}{2} a_{0}^{2} \mu_{\gamma}^{2}\right)
\end{aligned}
$$

Accounting for spin-spin coupling is identical to the Maxwell case, yielding,

$$
\Delta E_{h f}=\frac{2 g_{e} g_{p} e^{2} \hbar^{2}}{3 m_{e} m_{p} c^{2} a_{0}^{3}}\left(1-\frac{3}{2} a_{0}^{2} \mu_{\gamma}^{2}\right)=\frac{2}{3}\left(\frac{m_{e}}{m_{p}}\right) g_{e} g_{p} m_{e} c^{2} \alpha^{4}\left(1-\frac{3}{2} a_{0}^{2} \mu_{\gamma}^{2}\right)
$$

Thus, the first order correction to the $21 \mathrm{~cm}$ radiation to account for a massive photon is proportional to the square of the ratio between the photon mass and the photon Compton wavelength. With the current best experimental upper limit of $m_{\gamma} \sim 10^{-49} \mathrm{~g}$, this puts the correction term on the order of $10^{-40}$. Although this effect is likely too small to be detected with the current spectroscopic technology, it is possible to increase the likelihood by considering artificial atoms of larger dimensions (e.g. the Rydberg atoms 
[22]), or by exploring potential techniques in the enhancement of the sensitivity of various spectroscopic measurements.

Of note, the photon mass could also have non-trivial effects on the g-factors [23]. Also, to obtain the first order correction to the wavelength of the emitted radiation, the full dispersion relation must be used to the appropriate level of approximation. 
It is our hope that the theoretical results obtained here-in will yield further theoretical and experimental contributions to the field of massive electrodynamics. The aim of the brief summary of Chapters 2-4 is to put into context the motivation for the current work. The primary results obtained are, for a massive photon, a revision to Snell's Law (5.8), Fresnel's formulas (5.11) \& (5.12), and the changes to the $21 \mathrm{~cm}$ radiation (6.29). 
[1] A. Proca, "Sur la Théorie du Positron," Acad. Sci., Paris, C. R., vol. 202, pp. 1366-8, 1936.

[2] A. Proca, "Sur la Théorie Ondulatoire des Electrons Positifs et Négatifs," J. Phys. Radium, vol. 7, pp. 347-53, 1936.

[3] A. Proca, "Sur les Photons et let Particules Charge Pure," Acad. Sci., Paris, C.R., vol. 203, pp. 709-11, 1936.

[4] A. Proca, "Particles Libres: Photons et Particules Charge Pure," J. Phys. Radium, vol. 8, pp. 23-8, 1937.

[5] A. Proca, "Théorie Non Relativiste des Particules a Spin Entier," J. Phys. Radium, vol. 9, pp. 61-6, 1938.

[6] H. Yukawa, S. Sakata and M. Taketani, "On the Ineraction of Elementary Particles III," Proc. Phys. Math. Soc. Jpn., vol. 20, pp. 319-40, 1938.

[7] L. Tu, J. Luo and G. Gillies, "The Mass of the Photon," Rep. Prog. Phys., vol. 68, pp. 77-130, 2005.

[8] A. Goldhaber and M. Nieto, "Photon and Graviton Mass Limits," Rev. Mod. Phys., vol. 82, pp. 939-79, 2010.

[9] J. Jackson, Classical Electrodynamics 3rd Ed., John Wiley \& Sons, Inc., 1999.

[10] G. Barton and N. Dombey, "The Casimir Effect with Finite-Mass Photons," Ann. Phys., vol. 162, pp. 231-72, 1985.

[11] L. Teo, "Casamir Effect of Massive Vector Fields," Phys. Rev. D, vol. 82, p. 105002, 2010.

[12] P. Dragulin and P. Leung, "Green Dyadic for the Proca Fields," Phys. Rev. E, vol. 78, p. $026605,2008$.

[13] S. L. Garavelli and F. A. Oliveira, "Analytical Solution for a Yukawa-Type Potential," Phys. Rev. Lett., vol. 66, no. 10, pp. 1310-3, 1991. 
[14] E. Stueckelberg, "Théorie de la radiation de photons de masse arbitrairement petite," Helv. Phys. Lett., vol. 30, pp. 209-15, 1957.

[15] R. Thompson, S. Cummer and J. Frauendiener, "Generalized Transformation Optics of Linear Materials," J. Opt., vol. 13, p. 055105, 2011.

[16] N. Kroll, Phys. Rev. Lett., vol. 26, pp. 1395-8, 1971.

[17] H. Cavendish, Electrical Researches, J. Maxwell, Ed., Cambridge: Cambridge University Press, 1879, pp. 104-113.

[18] M. Chernikov, C. Gerber, H. Ott and H. Gerber, Phys. Rev. Lett., vol. 68, pp. 3383-6, 1992.

[19] R. Penrose, The Road to Reality: A Complete Guide to the Laws of the Universe, Vintage Books, 2004, pp. 410-1.

[20] Taylor and Mohr, "NIST Reference on Constants, Units, and Uncertainty," NIST Physical Measurement Laboratory, November 2012. [Online]. Available: http://physics.nist.gov/constants. [Accessed 27 May 2013].

[21] D. Griffiths, Introduction to Quantum Mechanics 2nd Ed, Prentice-Hall Inc., 2005.

[22] T. Gallagher, Rep. Prog. Phys., vol. 51, p. 143, 1988.

[23] G. Spavieri, J. Quintero, G. Gillies and M. Rodrigues, Eur. Phys. J. D., vol. 61, p. 531, 2011. 